\title{
Mechanical Implementation of Kinematic Synergy for Continual Grasping Generation of Anthropomorphic Hand
}

\author{
Wenbin Chen, Member, IEEE, Caihua Xiong, Member, IEEE, and Shigang Yue, Member, IEEE
}

\begin{abstract}
The synergy-based motion generation of current anthropomorphic hands generally employ the static posture synergy, which is extracted from quantities of joint trajectory, to design the mechanism or control strategy. Under this framework, the temporal weight sequences of each synergy from pregrasp phase to grasp phase are required for reproducing any grasping task. Moreover, the zero-offset posture has to be preset before starting any grasp. Thus, the whole grasp phase appears to be unlike natural human grasp. Up until now, no work in the literature addresses these issues toward simplifying the continual grasp by only inputting the grasp pattern. In this paper, the kinematic synergies observed in angular velocity profile are employed to design the motion generation mechanism. The kinematic synergy extracted from quantities of grasp tasks is implemented by the proposed eigen cam group in tendon space. The completely continual grasp from the fully extending posture only require averagely rotating the two eigen cam groups one cycle. The change of grasp pattern only depends on respecifying transmission ratio pair for the two eigen cam groups. An illustrated hand prototype is developed based on the proposed design principle and the grasping experiments demonstrate the feasibility of the design method. The potential applications include the prosthetic hand that is controlled by the classified pattern from the bio-signal.
\end{abstract}

Index Terms-Anthropomorphic hand, design principle, kinematic synergy, mechanical implementation.

\section{INTRODUCTION}

$\mathbf{T}$ HE human hand has a large number of mechanical degrees of freedom (DoF), which offer great flexibility to perform skillful grasp and manipulation [1]. When a hand performs any complex task, all its finger joints are closely coordinated over time. It is a particular challenge to control each joint of an anthropomorphic hand to precisely replicate the kinematic and dynamic characteristic of the time-varying posture of human hand in a complex task [2]. The most

Manuscript received November 13, 2013; revised March 25, 2014; accepted May 18, 2014. Recommended by Technical Editor N. G. Tsagarakis. This work was supported in part by the National Basic Research Program of China (973 Program) under Grant 2011CB013301, in part by the National Science Fund for Distinguished Young Scholars of China under Grant 51025518, in part by the National Natural Science Foundation of China under Grant 51335004 and Grant 61301225, and in part by EU FP7 Projects HAZCEPT under Grant 318907. (Corresponding author: Caihua Xiong.)

W. Chen and C. Xiong are with the State Key Laboratory of Digital Manufacturing Equipment and Technology, School of Mechanical Science and Engineering, Huazhong University of Science and Technology, Wuhan 430074, China (e-mail: wbchen@hust.edu.cn; chxiong@hust.edu.cn).

$\mathrm{S}$. Yue is with the School of Computer Science, University of Lincoln, Lincoln, LN6 7TS, U.K. (e-mail: syue@ lincoln. ac.uk).

Color versions of one or more of the figures in this paper are available online at http://ieeexplore.ieee.org.

Digital Object Identifier 10.1109/TMECH.2014.2329006 complex point is how to generate so many channels of control signals to coordinate the joints. In many cases, only very limited channels are available, such as the prosthetic hand controlled by a bio-signal. Thus, under this situation, the functional versatility and motion controllability seem to be incompatible for the anthropomorphic hand.

Since an under-actuated mechanism can reach the tradeoff between the versatile function and a few input channels, designing an anthropomorphic hand based on the under-actuation principle attracts lots of attention and many types of underactuated mechanisms are proposed in the literature to address this issue. Some are based on linkages [3]-[5], some are based on tendon-actuated mechanisms [6]-[8], while others are based on flexible actuators [9]-[11]. Due to the self-adaptation to the object grasped, the under-actuated hands have well compliance in limited inputs [12]. However, an under-actuated hand cannot produce fruitful postures in free space because the finger joints are not independently controlled. Unlike the under-actuated mechanism, the dimensionality reduction based techniques in the literature toward finding a lower-dimensional representation of the original grasp data, which also provides a potential way to address this problem. The dimensionality reduction methods generally consist of nonlinear and linear types. The most recent representatives of nonlinear approaches include the Gaussian process latent variable model [13] and unsupervised kernel regression [14]. The most commonly used linear approach is principal component analysis (PCA). The principal component or synergy [15]-[22] observed from the human hand grasping activities couple all joints to form a specified static or timevarying posture. Any posture or continual time-varying posture can be approximated by a linear combination of a few significant synergies. Compared with the nonlinear approaches, the PCA method is more feasible to be implemented by the real-time algorithm [23]-[27] and drive mechanism [28]-[30].

In the literature, much work has been done in applying the principle of synergy to deal with motion generation of anthropomorphic hands via a few inputs. These inputs are the weight for combining several static posture synergies. For example, two [23] and three [24] postural synergies are used to control 24 actuators in the ACT hand. Other examples include the use of two synergies in the DLR II hand [25], four synergies in the SAH hand [26], two synergies in the UB Hand IV [27], etc. Matrone et al. use the $X-Y$ coordinates of mouse cursor in screen [31] or two channels of sEMG from human wrist [32] as two control-inputs for the CyberHand. Different from the software regulation in the aforementioned works, 
the posture synergies were implemented via a designed mechanism in tendon space [28].

The common characteristic of the motion generation of these anthropomorphic hands is that the used posture synergy is static and has no temporal characteristics. Thus, the implementation of a time-varying posture for a particular grasping movement is dependent on carefully specifying the time-varying weights of each synergy. The general procedure for determining the value of weights for a particular grasping movement is that: first, adjust the hand to the zero-offset posture in which the weights of each synergy equal to zero. Second, open the hand to the fully extension posture. Third, close the hand to the suitable grasping posture. Finally, compute the corresponding weights at the two boundary postures. The intermediate temporal values of weight for this grasping movement are solved by linearly interpolating the weights corresponding to these three postures assuming appropriate time interval [27]. The hand should be in the zero-offset posture before starting any grasping movement. The temporal weights from simple interpolation cannot ensure that it precisely duplicates the practical values, which affects the hand's performance. Moreover, the temporal sequence of weights depend on the prespecified grasp configuration for the considered object, which affects the hand's performance in an unstructured environment. In most cases, what can be provided before grasp is the grasp pattern but not the final grasp configuration. Thus, the continual motion generation based on static posture synergy requires well-defined conditions that may limit its potential application.

To address these issues, we investigate mechanical implementation of the angular velocity synergy and propose general design principle of an eigen cam group to implement such type of synergy. The mechanical implementation of synthesizing and regulating synergic velocity are also studied. Based on the design principle, we propose a prototype of anthropomorphic hand, as shown in Fig. 1, whose continual grasp from initial posture to the final grasp posture only require averagely rotating the two input shafts one cycle under particular transmission ratio.

The major contribution of this paper is the first presentation of the design principle of mechanically implementing the angular velocity synergy, i.e., kinematic synergy, and the continual grasping movement is only dependent on the transmission ratio pair that can be considered as grasp pattern in practical applications. The tough procedure to compute the temporal weight sequence of each synergy for different grasp tasks is eliminated, and the presetting zero-offset posture before any grasp is not required any more. Thus, the control complexity is dramatically reduced. The most attractive advantage of the proposed method is that the whole period of continual motion is fully governed by a discrete signal, which is the potential to design a prosthetic hand that is controlled by a pattern signal decoded from the sEMG.

The rest of this paper is organized as follows. In Section II, mathematical background about the angular velocity synergy and its application in motion approximation is introduced. The experiment paradigm for synergy extraction and statistical analysis are given in Section III. In Section IV, the method of

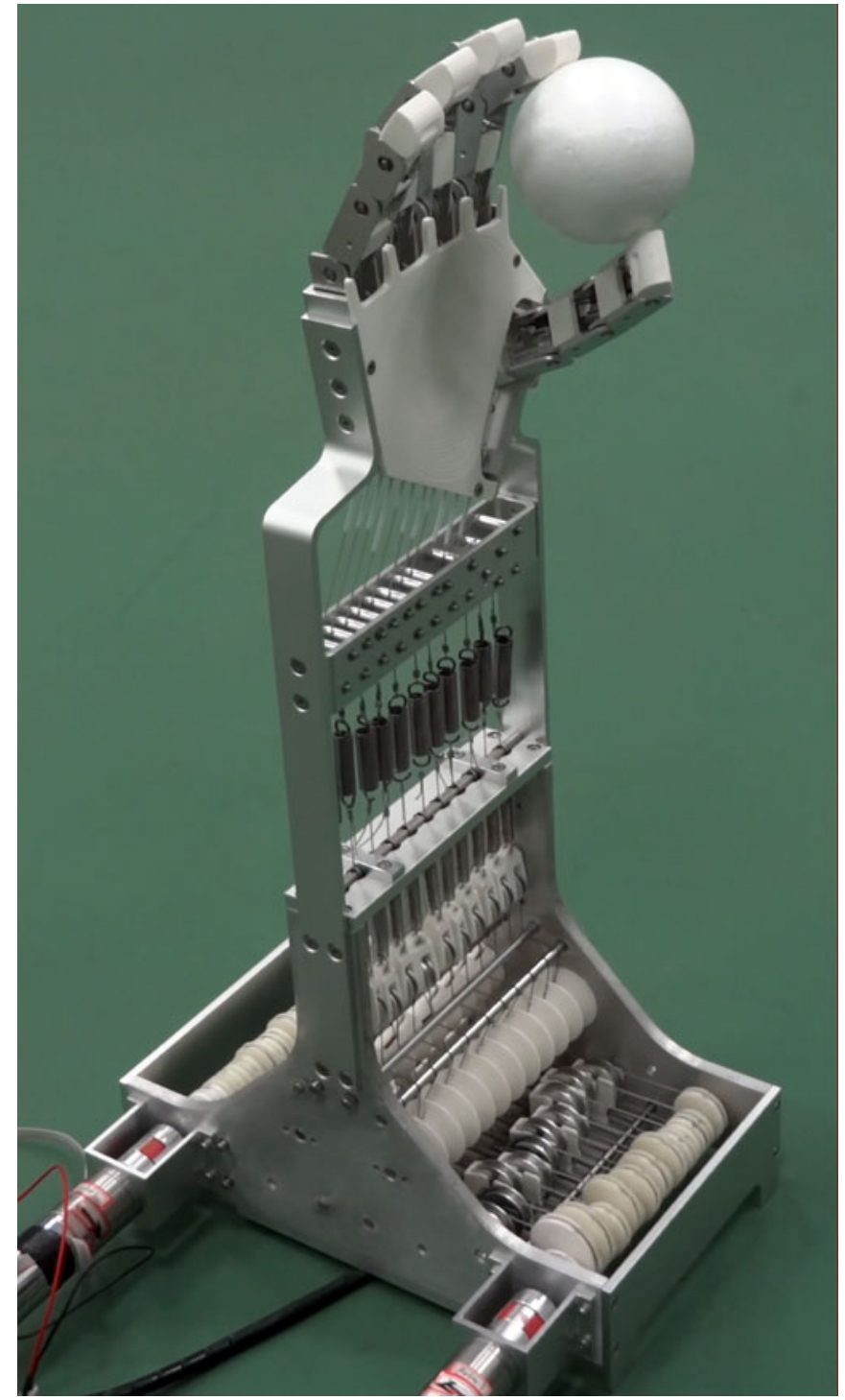

Fig. 1. Prototype of anthropomorphic hand based on the proposed design principle in this paper.

mechanism design is represented to implement the angular velocity synergy. A prototype of an anthropomorphic hand is developed based on the proposed method. The motion simulation and demonstration of grasping experiment are given in Section V. The discussion and conclusion are presented in Sections VI and VII, respectively.

\section{ANGULAR VELOCITY SyNERGY}

Before giving the detail of the design method, we begin with a brief overview of the mathematical form of angular velocity synergy. If $n$ joints are considered and the period of grasp or manipulation is averagely sampled $t_{s}$ times in one trial, the angular velocity evolvement of the hand joints at the duration can be represented as

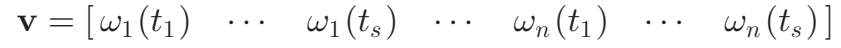


where the element $\omega_{i}\left(t_{j}\right)$ is the $i$ th joint velocity at the $t_{j}$ th sample $t_{1}$ represents the first sample at initial time and $t_{s}$ represents the final sample at end time). Given $N$ trials for grasping or manipulating of different objects, a velocity matrix can be defined by

$$
\mathbf{V}=\left[\begin{array}{c}
\mathbf{v}_{1} \\
\vdots \\
\mathbf{v}_{N}
\end{array}\right]
$$

where $\mathbf{v}_{i}(i=1 \cdots N)$ is the $i$ th trial of grasping or manipulation. This joint velocity matrix can be rewritten as product of three smaller matrices by singular value decomposition (SVD), which is illustrated by (3). Each row of the third matrix $\mathbf{S}$ is called a principal component (or called a synergy). The singular values are diagonally arranged from largest to smallest in second matrix

$$
\mathbf{V}=\mathbf{U} \operatorname{diag}\left\{\lambda_{1} \cdots \lambda_{m}\right\} \mathbf{S} .
$$

According to the importance of elements of the diagonal matrix, the matrix $\mathbf{V}$ can be approximated by $\hat{\mathbf{V}}$ if the first $k$ principal components account for more than $80 \%$ of the total variance of the entire trial data [15]

$$
\mathbf{V} \approx \hat{\mathbf{V}}=\mathbf{W}^{k} \mathbf{S}^{k}
$$

where $\mathbf{W}^{k}=\mathbf{U}_{k} \operatorname{diag}\left\{\lambda_{1} \cdots \lambda_{k}\right\}, \mathbf{U}_{k}$ is the first $k$ columns of matrix $\mathbf{U}$, and $\mathbf{S}^{k}$ is the first $k$ rows of matrix $\mathbf{S}$. In fact, according to our experimental data from human grasping movement, seeing the next section, the first two rows of the matrix $\mathbf{S}$ can account for $88 \%$ of the total variance, which is consistent with the statistical analysis result in [18]. We think these two principal components are sufficient to account for the variance of the entire trial data. Thus, the (4) can be written as

$$
\begin{aligned}
& \mathbf{V} \approx \mathbf{W}^{2} \mathbf{S}^{2}= \\
& {\left[\begin{array}{cc}
w_{1}^{1} & w_{1}^{2} \\
\vdots & \vdots \\
w_{q}^{1} & w_{q}^{2} \\
\vdots & \vdots \\
w_{N}^{1} & w_{N}^{2}
\end{array}\right]\left[\begin{array}{lllllll}
s_{1}^{1}\left(t_{1}\right) & \cdots & s_{1}^{1}\left(t_{s}\right) & \cdots & s_{n}^{1}\left(t_{1}\right) & \cdots & s_{n}^{1}\left(t_{s}\right) \\
s_{1}^{2}\left(t_{1}\right) & \cdots & s_{1}^{2}\left(t_{s}\right) & \cdots & s_{n}^{2}\left(t_{1}\right) & \cdots & s_{n}^{2}\left(t_{s}\right)
\end{array}\right]}
\end{aligned}
$$

where the scalars of $w_{i}^{1}$ and $w_{i}^{2}$ corresponding to the $i$ th row of matrix $\mathbf{V}$ are weights associated with the two principal components. Each principal component, i.e., the angular velocity synergy, is averagely sampled $t_{s}$ times. To further explicit representation, each row of $\mathbf{V}$, i.e., $\mathbf{v}_{i}$, corresponding to one trial can be written as

$$
\mathbf{v}_{i} \approx\left[\begin{array}{ll}
w_{i}^{1} & w_{i}^{2}
\end{array}\right] \mathbf{S}^{2}
$$

or rewritten in following form:

$$
\begin{aligned}
& {\left[\begin{array}{ccc}
\omega_{1}\left(t_{1}\right) & \cdots & \omega_{1}\left(t_{s}\right) \\
& \vdots & \\
\omega_{n}\left(t_{1}\right) & \cdots & \omega_{n}\left(t_{s}\right)
\end{array}\right]_{i}} \\
& \approx w_{i}^{1}\left[\begin{array}{ccc}
s_{1}^{1}\left(t_{1}\right) & \cdots & s_{1}^{1}\left(t_{s}\right) \\
& \vdots & \\
s_{n}^{1}\left(t_{1}\right) & \cdots & s_{n}^{1}\left(t_{s}\right)
\end{array}\right]+w_{i}^{2}\left[\begin{array}{ccc}
s_{1}^{2}\left(t_{1}\right) & \cdots & s_{1}^{2}\left(t_{s}\right) \\
& \vdots & \\
s_{n}^{2}\left(t_{1}\right) & \cdots & s_{n}^{2}\left(t_{s}\right)
\end{array}\right]
\end{aligned}
$$

where each row of the matrices at the two sides of (7) corresponds to one joint velocity evolvement at the duration of the hand movement. The reduced form of (7) for the $i$ th joint is given by

$$
\left.\left.\omega_{i}\right|_{t_{1}} ^{t_{s}} \approx w_{i}^{1} s_{i}^{1}\right|_{t_{1}} ^{t_{s}}+\left.w_{i}^{2} s_{i}^{2}\right|_{t_{1}} ^{t_{s}} \quad i=1, \ldots, n
$$

Although the elements of each angular velocity synergy are discretely represented in (7) due to discrete sampling, it must be noted that each element is a continually varying function of time. This characteristic of velocity synergy is different from the static posture synergy whose element is a constant scalar value. The static posture synergy just represents the principal motion direction in the joint space, and it cannot describe how to coordinate joints across time. The velocity synergy represents the eigen evolvement along the motion time. It just needs to specify one single weight for each velocity synergy to construct the continual time-varying posture for a complete manipulation. When initial hand posture is specified as the naturally fullextended fingers, the hand posture at each time during the motion period can be obtained by integrating angular velocity synergy.

In this paper, we aim to develop the design method toward reducing the control complexity and minimizing the quantity of input to implement the continual grasping movement. In the following sections, we will show how to achieve this goal by implementing the angular velocity synergy described by (7) in a mechanical manner.

\section{KinEMATIC SYNERGY EXTRACTION}

Here, we use the CyberGlove II to record the joints angle of the human hand during grasping objects with different shapes. Three healthy right-hand subjects wearing the CyberGlove are instructed to grasp each object shown in Fig. 2 for three times in appropriate grasp patterns. The initial posture of each grasp is with fully extended fingers and the abduction between the thumb and index finger is in the comfortable status (see Fig. 2). Each grasping movement is averagely performed in $3 \mathrm{~s}$ including one second for the start and one second for the end of the grasp to ensure the zero velocity at the two ends. The involved grasp patterns follow the grasp taxonomy proposed by Cutkosky [33]. In our experiment, each object is grasped in different feasible types as many as possible. Finally, there are 322 grasping movements performed in total. Here, we only consider eleven sensors that correspond to the thumb rotation, metacarpophalangeal (MCP), 


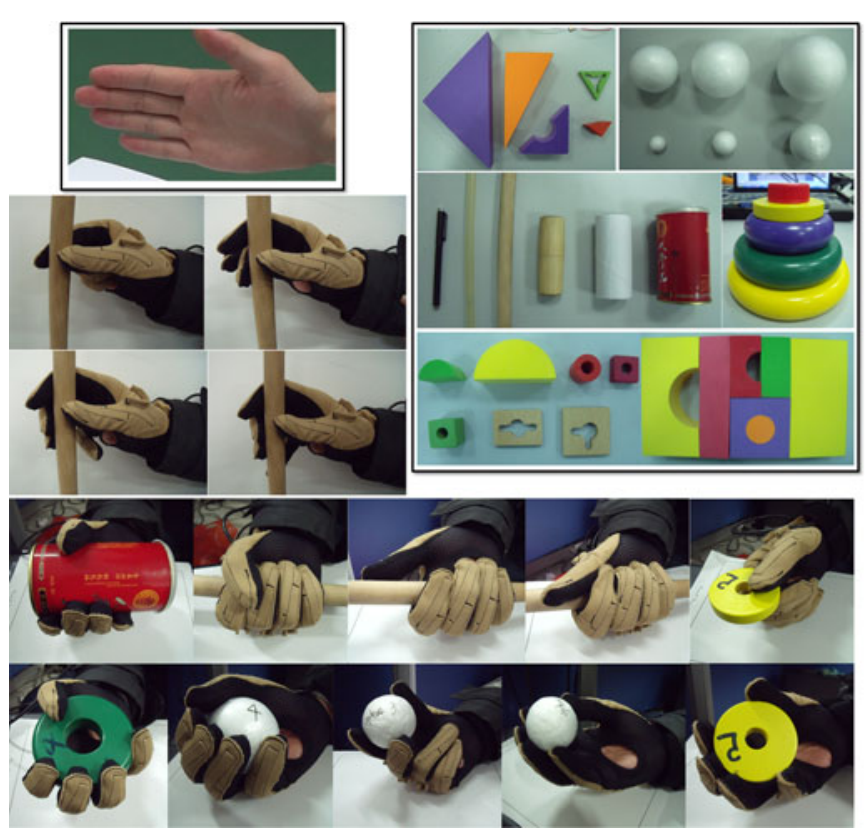

Fig. 2. Initial posture of each grasping movement (at left upper corner), the involved objects for grasping (at right corner), and the typical grasp patterns summarized in [33].

and interphalangeal joints of the thumb, and the $\mathrm{MCP}$ and proximal interphalangeal (PIP) joints of the other four fingers. These eleven joints can capture most characteristics of the human hand in grasping tasks. Note that the distal interphalangeal (DIP) joints are not considered because the flexion of the DIP joint of a finger is about two-thirds of that of the PIP joint [34].

Due to the resolution limit of the glove sensor, the raw trajectory of each joint shows a step profile that it is not suitable for derivative operation. All the raw data from the glove sensors are first fitted by a third-order spline with boundary constraints. Then, the resampled data are filtered using a low-pass Butterworth filter at a cut-off frequency of $2 \mathrm{~Hz}$. Finally, the excess stationary region at start and end stage is truncated and only the interval between onset time and mainly stopping time is reserved. Then, the motion time is normalized to one second for velocity derivation.

The velocity synergies are extracted by applying the SVD procedure to the derived velocity data. Upon this result, the first and second synergies account for approximately $80 \%$ and $88 \%$ of the total variance, respectively. Fig. 3 illustrates how sufficiency of synergy numbers account for the total variance of the entire training data. Error bars indicate standard deviation across subjects. The sufficiency of the synergy number is similar to the statistical result in [18]. Although the first three or more synergies can give more precision to account for the total variance, mechanically implementing more synergies will dramatically increase mechanism complexity comparing with mechanically implementing two synergies. This is one of the points where we only employ the first two synergies to replicate the grasping movement. The waveforms of the first two synergies are shown in Fig. 5.

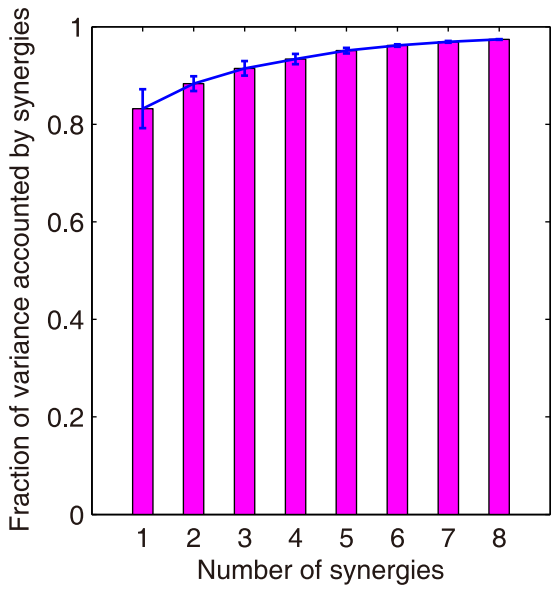

Fig. 3. Fraction of variance illustrating in bars is accounted by increasing number of synergies. Error bars indicate the standard deviation across subjects.

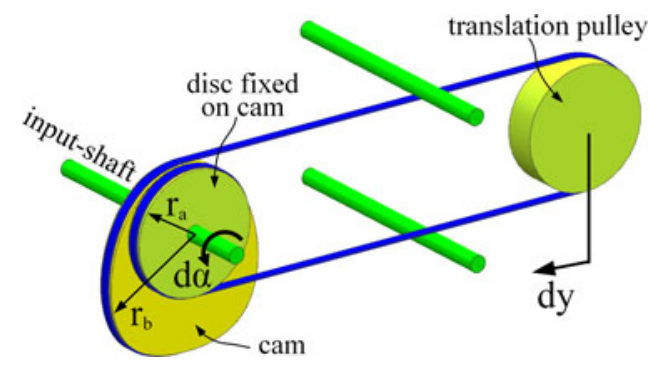

Fig. 4. Cam mechanism generating time-varying translation in tendon space. The axes of disc and cam are aligned. Notice that there is only one cable in this mechanism which two end-tips of the cable are fixed on cam and disc, respectively.

\section{Mechanism Design}

\section{A. Transmission Mechanism Design}

1) Cam Design Principle: The angular velocity profile of the element in one synergy is realized by a combined mechanism consisting of a cam, disc, and pulley. As shown in Fig. 4, the cam and disc are fixed together. The radius of the cam is the function of the rotational angle. The radii of cam and the disc at initial rotational angle are equal. When the input-shaft rotates at constant angular velocity, the differential motion between the input angle $d \alpha$ and output translation $d y$ has the following relationship:

$$
r_{b}(\alpha) d \alpha-r_{a} d \alpha=2 d y .
$$

Here, the $d y$ is described in tendon space and it can map to joint space just by winding a cable around the axis of a revolutionary joint. In this section, we call the revolutionary joint design-reference-finger-joint whose radius and differential rotation are denoted by $r_{s}$ and $d \alpha_{s}$ respectively. If the output translation $d y$ in tendon space is applied to the design-referencefinger-joint, the $d y$ can be represented as $d y=r_{s} d \alpha_{s}$. After taking it into (9), we obtain

$$
r_{b}(\alpha) d \alpha-r_{a} d \alpha=2 r_{s} d \alpha_{s}
$$




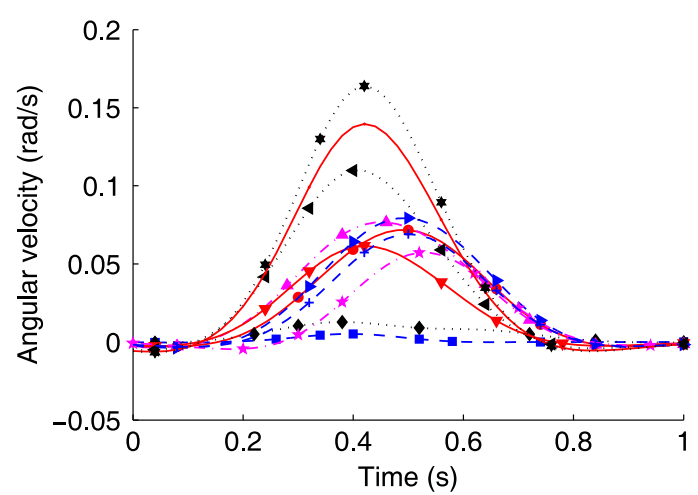

(a)

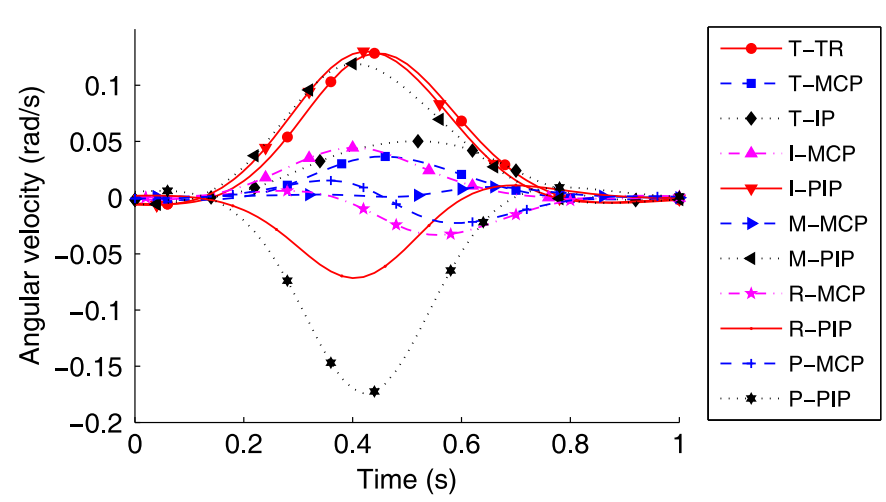

(b)

Fig. 5. Two most significant angular velocity synergies extracted from grasping trials. (a) the first significant angular velocity synergy, (b) the second significant angular velocity synergy. The abbreviated letters T, I, M, R, and P correspond to thumb, index finger, middle finger, ring finger, and pinky finger, respectively.

and dividing both sides of (10) by differential time $d t$, the expression about the radius of cam is formulated as

$$
r_{b}(\alpha)=2 r_{s} \frac{s(t)}{\bar{\omega}(t)_{0 \mapsto \alpha}}+r_{a}
$$

where $s(t)=d \alpha_{s} / d t$ can be considered as the angular velocity of one joint in velocity synergy and $\bar{\omega}(t)_{0 \mapsto \alpha}=d \alpha / d t$ denotes the constant angular velocity of the input axis in the range between 0 and $\alpha$. In this section, we call $\bar{\omega}$ input-referencerotation. According to (11), if the radii of the cam's disc and design-reference-finger-joint, i.e., $r_{a}$ and $r_{s}$, are specified, respectively, the radius of cam about the rotation angle $\alpha$ is particularly determined. This cam mechanism will ensure the velocity profile of design-reference-finger-joint to follow one waveform of the velocity synergy when the input-shaft rotates angle $\alpha$ from 0 radian at constant velocity $\bar{\omega}$ in one second. Because the velocity amplitudes in velocity synergy are generally small, the $r_{s}$ is not the real radius of finger joint but more likely the scaling coefficient of the radius. In order to simplify the problem, the values of the $r_{a}$ for all components of synergy are assumed to be same. Similarly, the values of the $r_{s}$ for all components of synergy are also assumed to be same. Under these assumptions, the cam radius at angle $\alpha$ is dependent on the magnitude of the corresponded component of synergy at time $t$ when the input axis rotates to angle $\alpha$.

Considering that the contour of cam should be continually smooth, we assume that the finger joint flexes from initial angle to the target angle when the input-shaft averagely rotates one cycle in one second. This assumption means that the maximum rotational angle of the input-shaft is $2 \pi$ radian and the inputreference-rotation $\bar{\omega}$ is $2 \pi \mathrm{rad} / \mathrm{s}$. When input-shaft rotates back from $2 \pi$ radian, the hand joints return to their initial position by the torsional springs installed at finger joint axes. For example, if the radii of cam's disc $r_{a}$ and design-reference-finger-joint $r_{s}$ are set as $r_{a}=10 \mathrm{~mm}$ and $r_{s}=150 \mathrm{~mm}$, using the first angular velocity synergy shown in Fig. 5 as the values of $s$, the resulting radii of cam and associated disc for the last ten joints are given in Fig. 6.
2) Cam Contour Correction: Note that some components of the second angular velocity synergy, such as R-MCP, RPIP, P-MCP, and P-PIP, have obviously minus velocity profile which will result in the corresponding cam with concave contour according to the design formula (11) [see Fig. 7(a)]. Because the concave contour brings noncontinual contact between the cable and cam surface, the outputted velocity profiles will not follow profiles of the synergy. This problem can be solved by rotating the cam $180^{\circ}$ around the axis that is parallel to the direction of translation of movable pulley, and then change the cable winding in reverse manner (see the exampled cam 3 in Fig. 8). The design formula for this type of cam is modified as follows:

$$
r_{b}^{\prime}(\alpha)=-2 r_{s} \frac{s(t)}{\bar{\omega}(t)_{0 \mapsto \alpha}}+r_{a}
$$

where $s(t)$ corresponds to the special component which has obviously minus profile, such as R-MCP, R-PIP, P-MCP, and P-PIP in the second synergy. The corrected contour of the cams corresponding to these four components of the second synergy are shown in Fig. 7(b).

3) Time-Varying Posture Synthesis: In this section, we will use the cam mechanism proposed in the previous section to synthesize the time-varying posture in velocity profile represented by (7). The mechanism principle of angular velocity synthesis for one joint is shown in Fig. 9. This mechanism can be decomposed into two parts according to function: synergic velocity generator and ratio regulator. The synergic velocity generator is the above-proposed cam mechanism. The ratio regulator is similar to a cylindrical cone and has continual varying diameter along axis. The 3-D solid model of the ratio regulator is given in Fig. 10. The diameter ratio of end section to other section is variable and can be viewed as amplifier to scale the velocity outputted from the synergic velocity generator, thus, further to regulate the outputted velocity of synthesis pulley. In following section, we call the diameter ratio of end section to other section transmission ratio. 

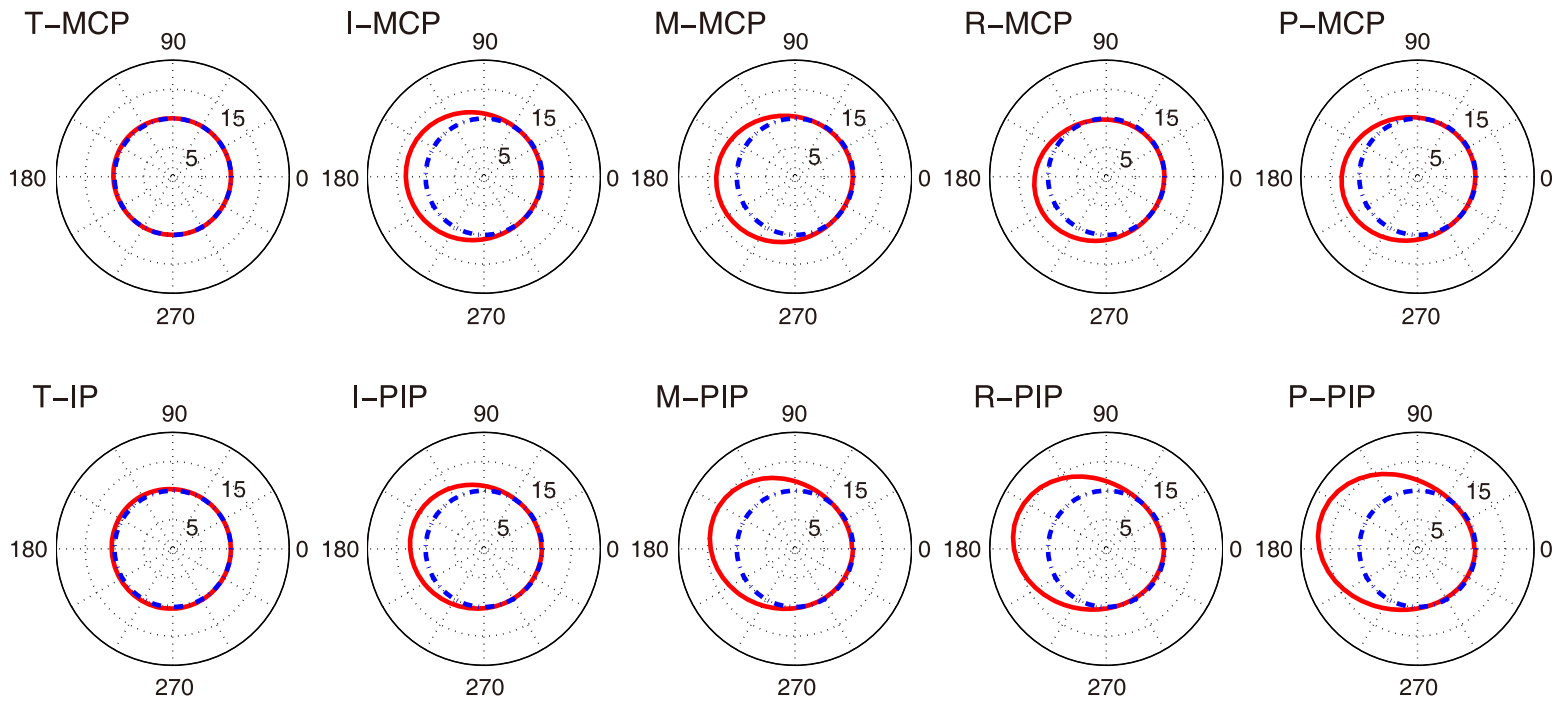

Fig. 6. Exampled cams and associated discs related to rotational angle in polar coordinate (radius-angle, unit: mm-degree) corresponding to the ten joints of the first kinematic synergy. The red solid curves represent the cam contour and the blue dash dot cycles represent the disc rigidly attached to cam.
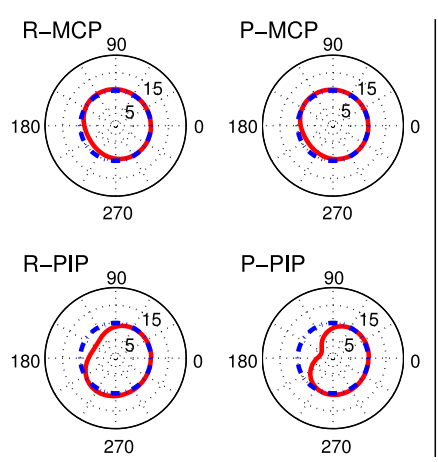

(a)
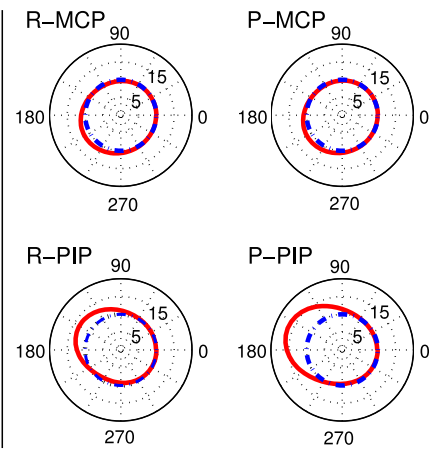

(b)

Fig. 7. Comparison of the cams contour in polar coordinate (radius-angle, unit: mm-degree) between (a) before correction and (b) after correction corresponding to the four components R-MCP, R-PIP, P-MCP, and P-PIP in the second synergy.

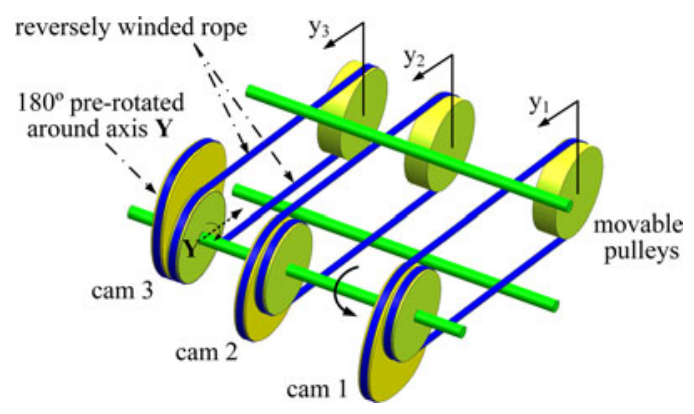

Fig. 8. Three exampled cams attach on the input-shaft. Notice that the cam 3 is prerotated $180^{\circ}$ around the $Y$-axis paralleling to the translation direction of movable pulley, which is to generate obviously minus velocity profile of component in velocity synergy.

As shown in Fig. 9, the output $d y_{i}$ for the $i$ th joint $(i=1, \ldots, 11)$ is given by

$$
K^{1}\left(r_{b i}^{1} d \alpha^{1}-r_{a}^{1} d \alpha^{1}\right)+K^{2}\left(r_{b i}^{2} d \alpha^{2}-r_{a}^{2} d \alpha^{2}\right)=4 d y_{i}
$$

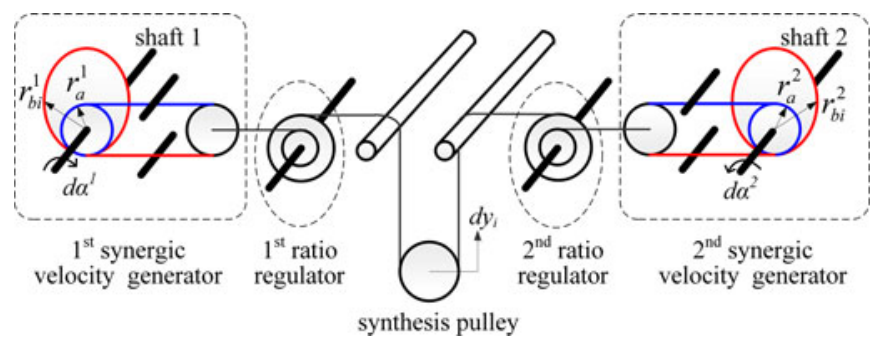

Fig. 9. Combining the two cam mechanisms to implement the kinematic synergy. The output velocity is synthesized in tendon space and can be transferred to the joint space through cable.

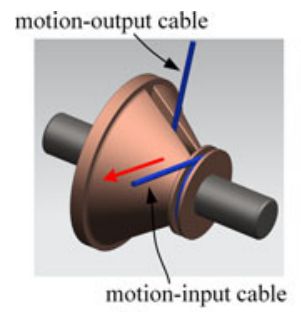

(a)

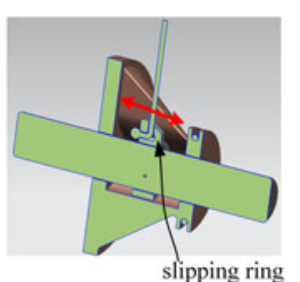

(b)

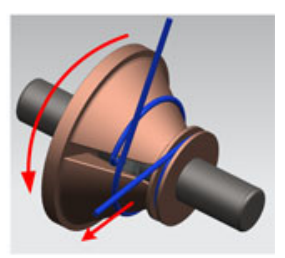

(c)
Fig. 10. 3-D model of the ratio regulator. (a) The initial configuration, (b) section view, (c) working configuration. The transmission ratio between the motion-input cable and motion-output cable can be freely adjusted by moving slipping ring on the axis when ratio regulator at initial configuration (corresponding to the initial hand posture).

where $K^{1}$ and $K^{2}$ are the transmission ratio corresponding to the first and second ratio regulator, respectively. $r_{b i}^{1}$ and $r_{b i}^{2}$ are the cam radii corresponding to the components of first and second velocity synergy, respectively. $r_{a}^{1}$ and $r_{a}^{2}$ are the radii of cam associated disc corresponding to cam $r_{b i}^{1}$ and $r_{b i}^{2}$, respectively. If we apply $d y_{i}$ to the $i$ th finger joint of the anthropomorphic hand, it can be reasonably formulated as $d y_{i}=r_{i}^{\circ} d \alpha_{i}^{\circ}$ in which $r_{i}^{\mathrm{o}}$ and $d \alpha_{i}^{\mathrm{o}}$ denote radius and differential rotation of the $i$ th finger 
joint, respectively. In order to simplify the velocity synthesis and focus on the motion analysis within the tendon space, we assume the radius of all the finger joints $r_{i}^{\circ}$ are same and equal to $r^{\circ}$. The (13) can be rewritten in the following form:

$$
\frac{K^{1} \omega^{1}}{4 r^{\circ}}\left(r_{b i}^{1}-r_{a}^{1}\right)+\frac{K^{2} \omega^{2}}{4 r^{\circ}}\left(r_{b i}^{2}-r_{a}^{2}\right)=\omega_{i}^{\mathrm{o}}
$$

where $\omega^{1}=d \alpha^{1} / d t, \omega^{2}=d \alpha^{2} / d t$, and $\omega_{i}^{\mathrm{o}}=d \alpha_{i}^{\mathrm{o}} / d t$. The $\omega^{1}$ and $\omega^{2}$ denote the rotational velocity of shaft 1 and 2 , respectively. The $\omega_{i}^{\mathrm{o}}$ denotes angular velocity of the $i$ th hand joint. According to the cam design principle in the previous section, the $\omega^{1}$ and $\omega^{2}$ are both equal to the input-reference-rotation $\bar{\omega}$, i.e., $2 \pi \mathrm{rad} / \mathrm{s}$. From (11), we have

$$
\begin{aligned}
& r_{b i}^{1}-r_{a}^{1}=2 r_{s}^{1} s_{i}^{1} / \bar{\omega} \\
& r_{b i}^{2}-r_{a}^{2}=2 r_{s}^{2} s_{i}^{2} / \bar{\omega}
\end{aligned}
$$

where $s_{i}^{1}$ and $s_{i}^{2}$ represent the $i$ th component of the first and second velocity synergy in (7), respectively. Similarly, the $r_{s}^{1}$ and $r_{s}^{2}$ are the radius of design-reference-finger-joint corresponding to the first and second velocity synergy, respectively. After putting them into (14), the angular velocity of $i$ th joint of hand is given by

$$
\omega_{i}^{\mathrm{o}}=\frac{r_{s}^{1}}{2 r^{0}} K^{1} \cdot s_{i}^{1}+\frac{r_{s}^{2}}{2 r^{0}} K^{2} \cdot s_{i}^{2}, \quad i=1, \ldots, 11 .
$$

Comparing (8) and (17), we basically implement the kinematic synergy synthesis via the proposed cam mechanism. To perform one particular grasping task from an initial fully extending posture, it only needs to specify the transmission ratio, i.e., $K^{1}$ and $K^{2}$, and then the two input-shafts rotate one cycle at speed of input-reference-rotation $\bar{\omega}$, i.e., $2 \pi \mathrm{rad} / \mathrm{s}$.

4) Design Parameters Optimization: In the previous sections, we have made three assumptions about the design parameters to simplify the mechanism design: The radii $r_{s}$ of design-reference-finger-joint corresponding to all components of one synergy are same, the radii $r_{a}$ of disc associated with cam corresponding to all components of one synergy are same, and the joint radii $r^{\circ}$ of all fingers are same. Changing $r_{s}$ and $r_{a}$ will affect the size of cams. Proper $r_{s}$ and $r^{\circ}$ can limit the transmission ratio in lower range which brings the ratio regulator in compact size. In order to limit the size of the cams and ensure the ratio regulator with compact size, a simple optimization procedure corresponding to each velocity synergy can be formulated as

$$
\begin{aligned}
& \min : f\left(r_{s}^{k}, r_{a}^{k}, r^{\circ}\right)=2 r_{s}^{k}\left|s_{i}^{k}\right|_{\max } /(2 \pi)+r_{a}^{k}, \quad k=1,2 \\
& \text { subject to : } \\
& 0<K^{k}=\left|w^{k}\right|_{\max } / \frac{r_{s}^{k}}{2 r^{\circ}} \leq \beta \\
& r_{\min }^{\mathrm{o}} \leq r^{\circ} \leq r_{\max }^{\mathrm{o}} \\
& r_{\mathrm{amin}} \leq r_{a}^{k} \leq r_{\max }
\end{aligned}
$$

the meaning of objective function is to limit the maximum radius of cam corresponding to the joint with most maximum velocity peak in kinematic synergy. The first constraint condition is to limit the transmission ratio in proper range so that size of the ratio regulator is compact.
TABLE I

VALUE OF BOUNDARY CONDITION FOR OPTIMIZATION

\begin{tabular}{lccc}
\hline \hline Boundary parameters & value & Boundary parameters & value \\
\hline$\beta$ & 2 & $r_{a \mathrm{max}}, r_{a \mathrm{~m} \mathrm{in}}$ & $15 \mathrm{~mm}, 10 \mathrm{~mm}$ \\
$r^{\circ}{ }_{\mathrm{max}}, r^{\circ}{ }_{\mathrm{min}}$ & $10 \mathrm{~mm}, 5 \mathrm{~mm}$ & & \\
\hline \hline
\end{tabular}

TABLE II

Optimal Result FOR THE CAM MECHANISM

\begin{tabular}{lcccc}
\hline \hline \multicolumn{2}{c}{ First kinematic synergy } & & \multicolumn{2}{c}{ Second kinematic synergy } \\
\cline { 1 - 1 } Parameters & Value & & Parameters & Value \\
\hline$r_{s}^{1}$ & $300 \mathrm{~mm}$ & $r_{s}^{2}$ & $102.5 \mathrm{~mm}$ \\
$r_{a}^{1}$ & $10.0 \mathrm{~mm}$ & & $r_{a}^{2}$ & $10.0 \mathrm{~mm}$ \\
$r^{0}$ & $5.0 \mathrm{~mm}$ & & $r^{\circ}$ & $5.0 \mathrm{~mm}$ \\
optimal value of & $24.8 \mathrm{~mm}$ & & optimal value of & $15.3 \mathrm{~mm}$ \\
objective function & & & objective function \\
\hline \hline
\end{tabular}

Before optimization, several parameters should be specified firstly. The maximum absolute peak in two kinematic synergies, i.e., $\left|s_{i}^{1}\right|_{\max }$ and $\left|s_{i}^{2}\right|_{\max }$, can be determined from the velocity synergies shown in Fig. 5 and here their value are found to be 0.16 and $0.17 \mathrm{rad} / \mathrm{s}$, respectively. The maximum absolute weight, i.e., $\left|w^{1}\right|_{\max }$ and $\left|w^{2}\right|_{\max }$, which can also be found from the SVD result of the motion capture data, are 56 and 18, respectively. Other boundary conditions are given in Table I. Using the boundary optimization procedure in MATLAB, the optimal results of the design parameters under given constraint are obtained (see Table II).

\section{B. Prototype Design}

According to the optimal result, the radii of the finger joints corresponding to the first and second synergy are the same value, which is consistent with the design assumption. The resultant cams can be assembled sequentially one by one on a common axis. These cams are fixed on the axis so that the grouped cams have same angular velocity. We call the grouped cams as the eigen cam group. According to the optimized parameters, the prototypes of the two eigen cam groups are shown in Fig. 11. In order to change the transmission ratio conveniently, the conoid surface of the ratio regulator along axis is separated into multigrooves whose radii are increased one by one. The improved ratio regulator and its assembly are shown in Fig. 12. The number of grooves are not optimized here, but just in the range that the selectable radius ratios can meet most of the grasp task. According to (17), changing the transmission ratio will scale the magnitude of the synergetic velocity outputted from the cam mechanism, which will alter the maximum translation of cable winding around the synthesis pulley. It must be noted that this maximum translation cannot be changed just by varying the rotation speed of the input shaft, because one cycle rotation of the cam fixes the output distance of movable pulley in the cam mechanism irregardless of the rotation speed.

The first version of the anthropomorphic hand prototype is developed, as shown in Fig. 13, for illustrating how to use the 


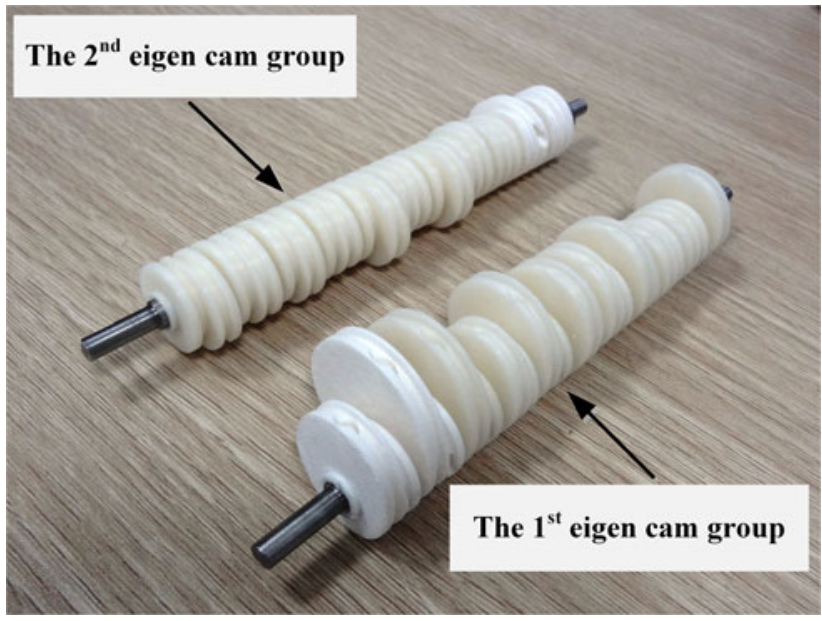

Fig. 11. Two eigen cam groups corresponding to the two angular velocity synergies in Fig. 5.

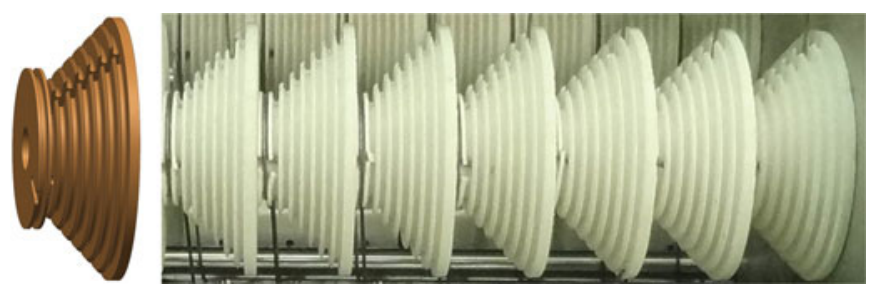

Fig. 12. 3-D solid model of the improved ratio regulator and local view of their assembly on a common axis.

two eigen cam groups to generate the continual grasping movement. Two local views are also provided to illustrate the main components, i.e., springs in the cable routers and the velocity synthesis mechanism. The detailed cable router in the drive mechanism are shown in Fig. 14. There are 15 joints in the current hand prototype with five similar fingers and each finger has three flexion/extension joints. The typical finger mechanism with cable router are shown in Fig. 15. The index, middle, ring, and pinky finger are all have two active joints (proximal and medial joint) and one coupled joint (distal joint). As shown in Fig. 15, the tendon driving the medial joint passes through the axis of proximal joint so that the coupled motion between medial and proximal joint due to winded cable is suppressed. The thumb has three active joints in which the coupled joint shown in Fig. 15 is replaced by an active one. The torsion springs with stiffness $0.36 \mathrm{Nmm} / \mathrm{deg}$ (model: T026-120-250 for medial and distal joint) and $0.65 \mathrm{Nmm} / \mathrm{deg}$ (T032-120-250 for proximal joint, SPEC Ltd) are mounted in the joint axis to guarantee the joint moving back when the cable is relaxed. The base of the thumb is fixed on the palm in an angle of $70^{\circ}$ to the palm from side view and $30^{\circ}$ to the palm from the top view. We find that this mounting manner is most appropriate for the thumb to perform precise grasping and power grasping in the current quantity of DoF. Under this framework, we map the rotation of human thumb to the flexion/extension of MCP joint of the robot thumb, which is similar to the joint mapping of CyberHand used in [35].

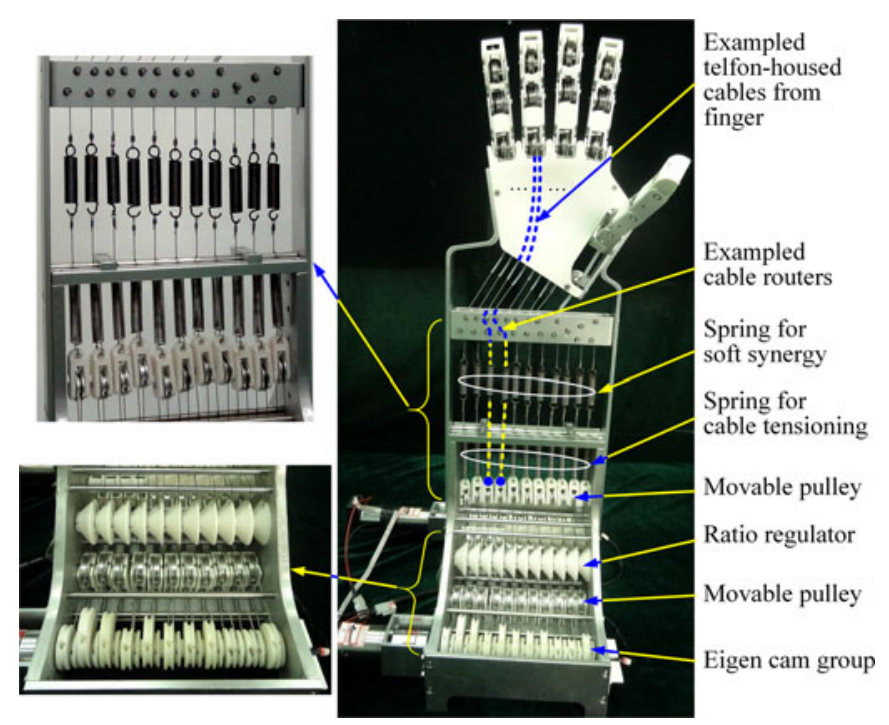

Fig. 13. Prototype of anthropomorphic hand based on the proposed principle of mechanical implementation of kinematic synergy. Two local views about the prototype are also given: the velocity synthesis mechanism and springs used to implement soft synergy and cables tensioning.

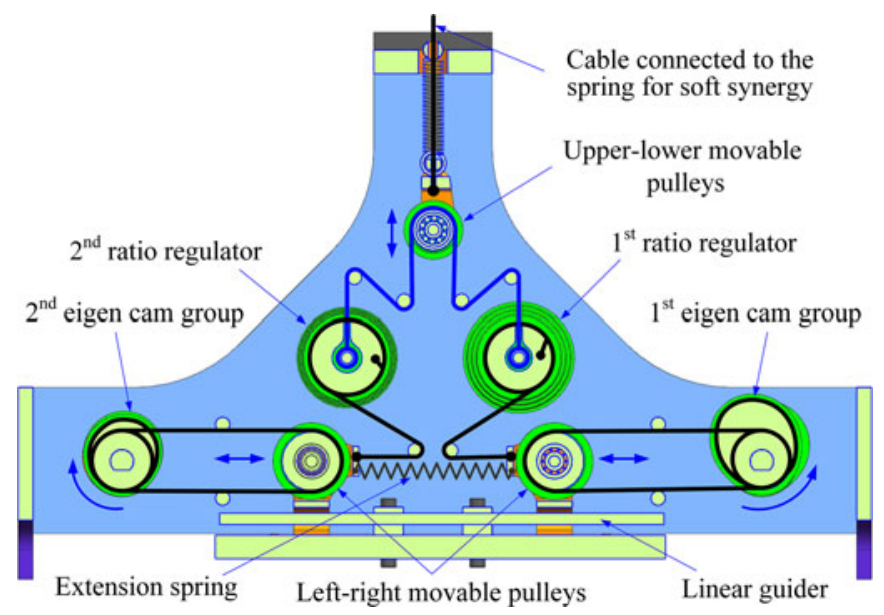

Fig. 14. Cable router in the velocity synthesis mechanism. The extension spring connected the left and right moveable pulleys is used to tension the cables winding around cam mechanism.

In order to assign compliant ability to the anthropomorphic hand, in case the final envelope formed by the hand posture is not consistent with the object shape, extension springs are placed into the tendon route between the fingers and the drive mechanism (refer to the springs for soft synergy in Fig. 12). The function of actuation mechanism is more like the conception of soft synergies which is first proposed in [36] and further developed in [37]. In fact, these extension springs are crucial to the appropriate grasp, because the drive mechanism is rigid and the stall of any finger joint during the input-shaft rotation will interrupt the motion of other joints, and even deform the grasped object if no tendons are broken in this case.

For the two types of spring in the cable router of the prototype, as shown in Fig. 13, the stiffness of the springs implementing 


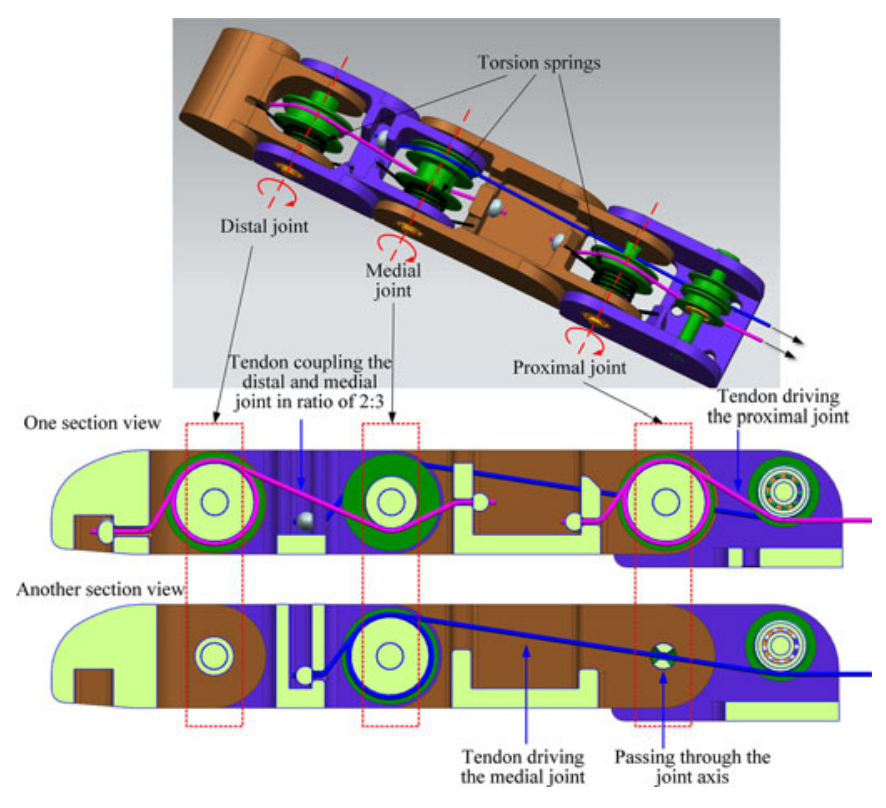

Fig. 15. Mechanism of one typical finger. Two section views are given to illustrate the involved cable routes.

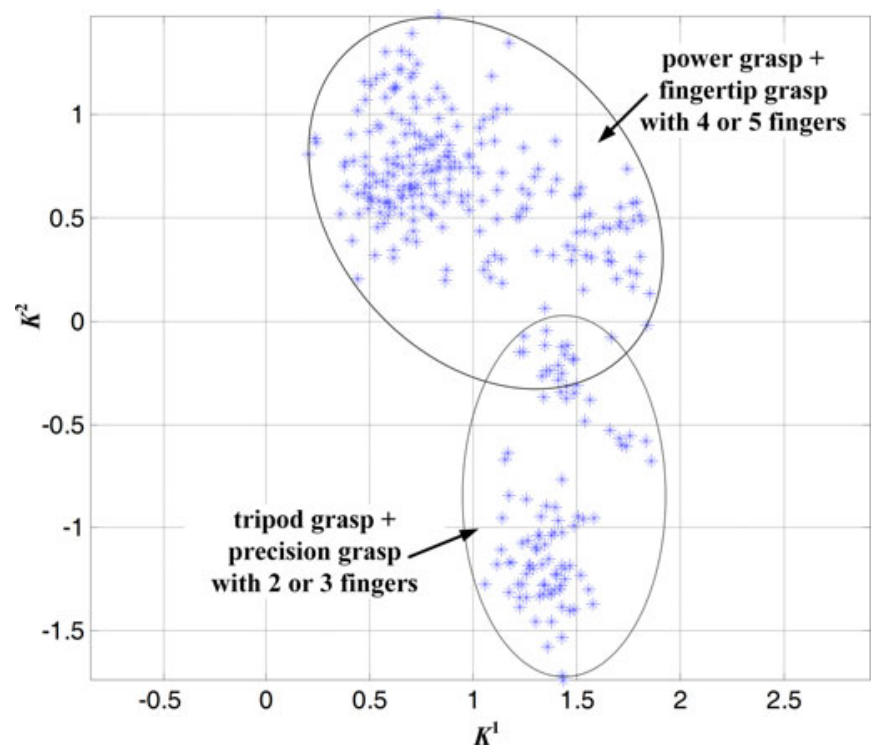

Fig. 16. Distribution of transmission ratio pair $\left(K^{1}, K^{2}\right)$. According to the final hand shape and involved fingers function, the transmission pairs are generally classified into two groups. The first one is called by power grasp and fingertip grasp with four or five fingers. These movements generally require almost all finger roughly flexed as enveloped shape to hold object without precision. The second one is called by tripod grasp and precision grasping with two or three fingers. These movements generally require the thumb, index, and optional middle finger to grasp object by fingertips.

the function of soft synergy are not optimized and uniformly specified as $4 \mathrm{~N} / \mathrm{mm}$ (model: E0360-055-1750M, SPEC Ltd.) just for the appropriate grasping force. The lower ends of these springs are connected to the upper-lower movable pulleys. The other type of springs for tensioning the cables are suspended at the shelf and the other ends are also connected to the upperlower movable pulleys. The stiffness of this type of spring is specified as $0.2 \mathrm{~N} / \mathrm{mm}$ (model: E0360-031-1750M, SPEC Ltd.). Considering the input-shafts have to provide enough torque to overcome the spring's extension force, the motor with nominal output torque 3.6 Nm (model: DBM32.60.36.100 (APE32), MOTEC Ltd.) is employed in the prototype.

According to the (8) and the (17), the transmission ratio is linearly proportional to the weight of the kinematic synergy. Based on weights that are computed from the SVD decomposition of experimental data, the distribution of transmission ratio pair $\left(K^{1}, K^{2}\right)$ are shown in Fig. 16. According to the trial data, the range of the ratio $K^{1}$ and $K^{2}$ is $(0,2)$ and $(-1.5,1.5)$, respectively. In the proposed hand prototype, the designed ranges for $K^{1}$ and $K^{2}$ are selected to be $(0.3,2)$ and $(0.3,1.5)$ considering the design feasibility of the ratio regulator. The minus value of $K^{2}$ in the range $(-1.5,-0.3)$ can be implemented by inversely rotating the second input-shaft. Thus, except for a small region that cannot be reached, the designed ratio regulator can approximate most of the real ratios.

In our experiment, the grasp task and its related objects can be generally defined as grasp pattern which follows the grasp taxonomy proposed by Cutkosky [33]. All the grasp trials performed by the same grasp pattern can be grouped together. The corresponding transmission ratio pairs grouped on plane can be represented by their average point. This average point, i.e., average transmission ratio pair, can be viewed as the typical representative for the grasp pattern. All typical transmission ratio pairs can be organized as grasp patterns and stored in the hand controller. In order to generate practical grasping movement, it is required only to specify the corresponding transmission ratio pair according to the grasp pattern and then averagely rotate the input-shafts one cycle.

The prototype in the current version does not replicate all possible joints of the human hand, such as the thumb rotation and abduction between fingers. This prototype is just for illustration of the implementation possibility of the continual motion generation of the robotic hand whose joints are driven by the eigen cam groups. If more DoFs are added to the prototype, more cams will be involved and the robotic hand can realize more comprehensive grasping movement.

\section{Simulation AND EXPERIMENT}

\section{A. Simulation Based on Synergy Synthesis}

In order to simply illustrate how the transmission ratio affects temporal evolvement of hand posture, here we select four typical transmission ratios for each synergy. The corresponded timevarying hand postures for each transmission ratio are averagely sampled along the motion time by five snapshots (see Fig. 17).

The first kinematic synergy mainly generates the power grasping movement. A bigger transmission ratio means more flexion posture. The second kinematic synergy mainly generates the precision grasp. Similarly, the flexion extent is in proportion to value of transmission ratio. Especially, the ring and pinky fingers inversely flex to the back of palm when the transmission ratio is positive, while the thumb, index, and middle finger flex inversely to the back of palm when the transmission ratio is negative. This phenomenon indicates that the first kinematic synergy 


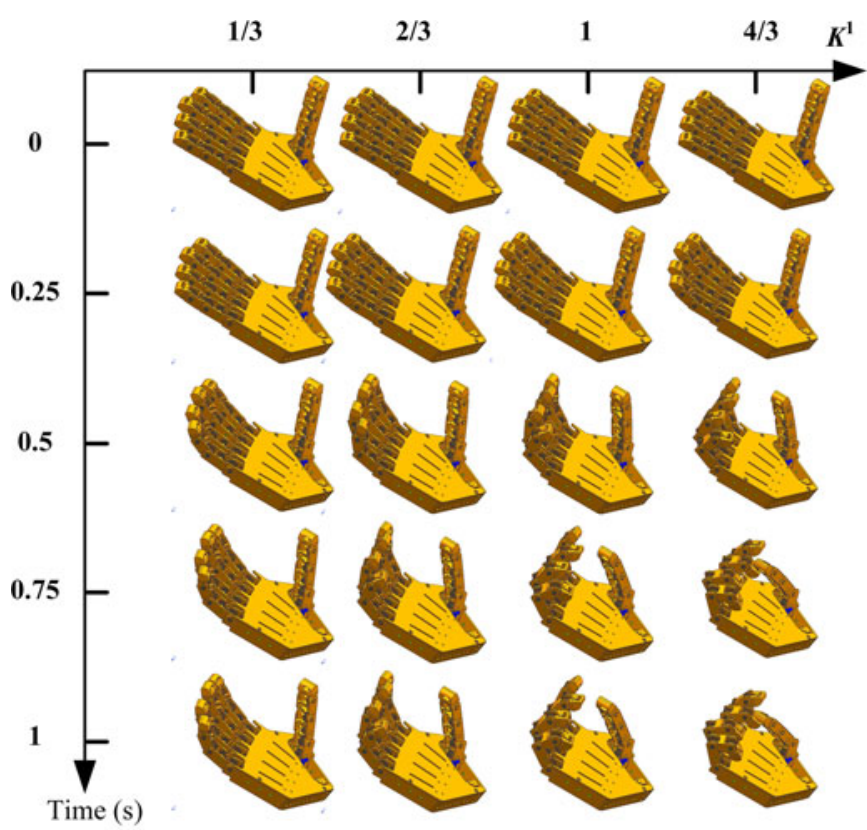

(a)

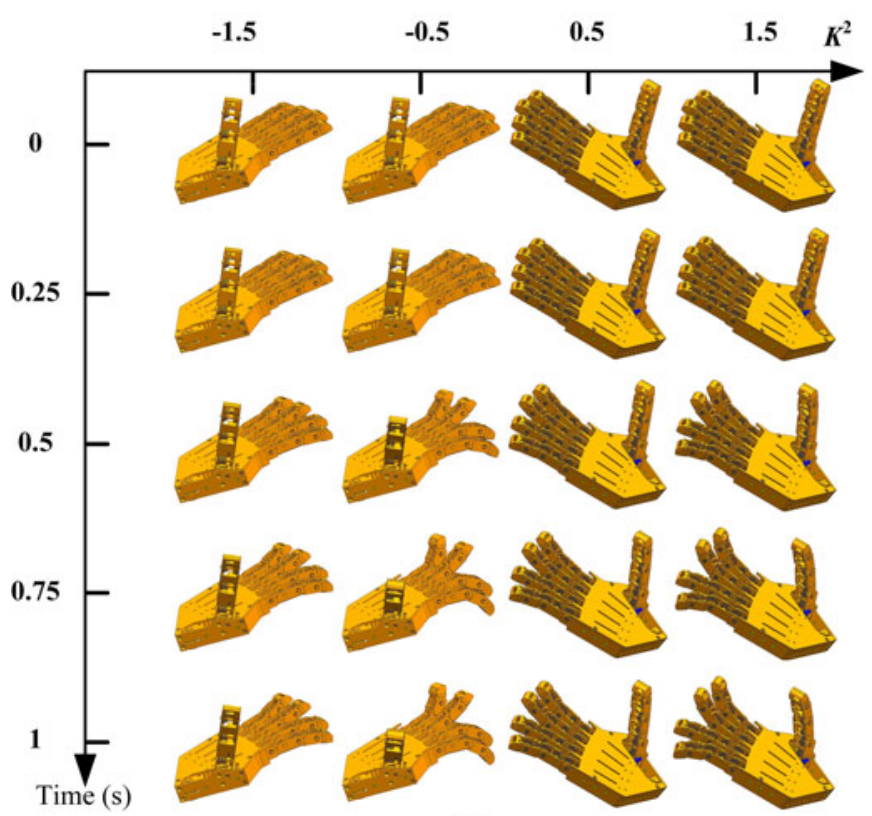

(b)

Fig. 17. Temporal sequence of hand postures at different transmission ratio corresponding to (a) the first synergy and (b) the second synergy, respectively.

plays the significant role in grasp posture formation, especially the power grasping movement, while the second kinematic synergy just counteracts part of the fingers flexion generated by the first kinematic synergy for producing complex movement, such as precision grasp. Due to mechanical constraints, no joint can inversely flex and thus there is no physical meaning to use the second velocity synergy alone. The rotation of second eigen cam group should be always in company with the rotation of first eigen cam group.

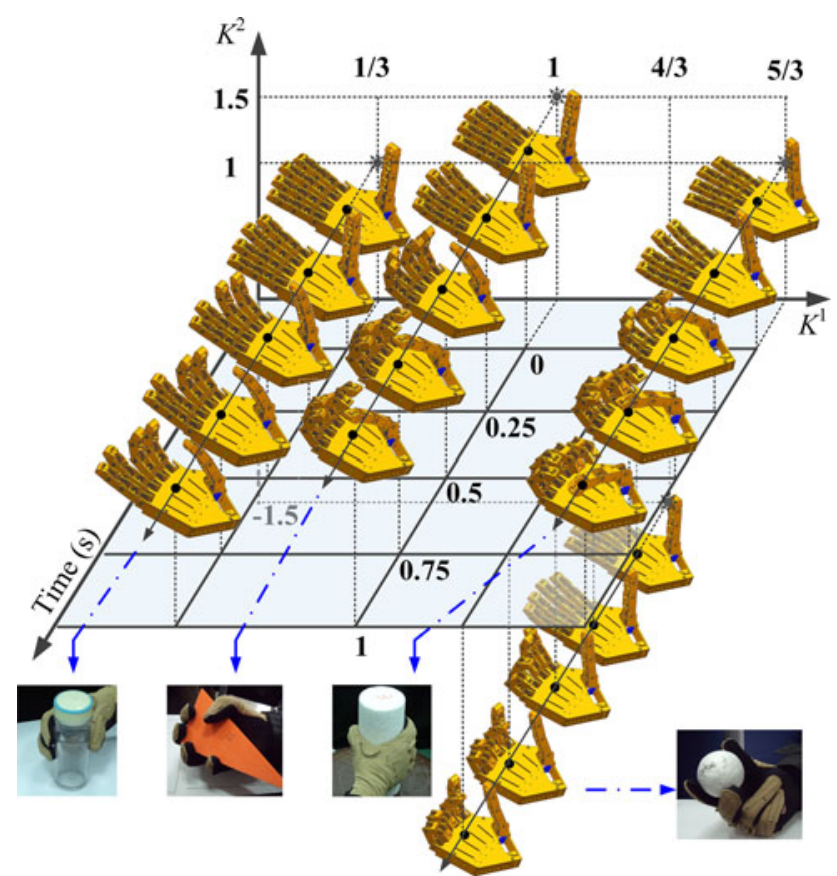

Fig. 18. Resultant temporal sequence of hand postures by combing the first and second synergy at different transmission ratios.

Via combining the two kinematic synergies under different weight (transmission ratio), practical grasping movement can be approximately generated. Fig. 18 gives four exampled resulting motions of the two kinematic synergies: grasping bottle with three fingers, power-grasping triangle, power-grasping cylinder, and precisely grasping sphere with three fingers. Due to the gap between robot thumb and human thumb on DoF distribution, the thumb movement of robotic hand is slightly different from that of human thumb, especially in the movement of power grasping cylinder. The joint trajectories for the movements under four transmission ratio pairs, i.e., $(4 / 3,-1.5),(1,1.5),(5 / 3,1)$, and $(1 / 3,1)$, are given in Fig. 21 . All joint ranges are mechanically limited in $\left[-8^{\circ}, 115^{\circ}\right]$, and the joint whose synthesis motion over this limitation will halt (see the P-PIP and M-PIP joint in Fig. 21(a) and (c), respectively).

As shown in Fig. 21, different combinations of transmission ratios of the two kinematic synergies produce generally different hand grasp patterns. Although more complex grasping movements can be reproduced by using more kinematic synergies [18], it will dramatically increase complexity on mechanism implementation.

\section{B. Grasping Experiments by the Prototype}

Here, we select three grasping tasks to validate the continual grasping ability: grasping a sphere, grasping a triangle, and grasping a cylinder. The initial posture for all tasks is the fingers in full extension. The transmission ratio pair of the three tasks are set to $(4 / 3,-1.5),(1,1.5)$, and $(5 / 3,1)$, respectively. The motion time is one second. When the two input motors averagely rotate one cycle, the temporal postures reach to the final grasp configuration. Like the illustration in the aforementioned 
(a)
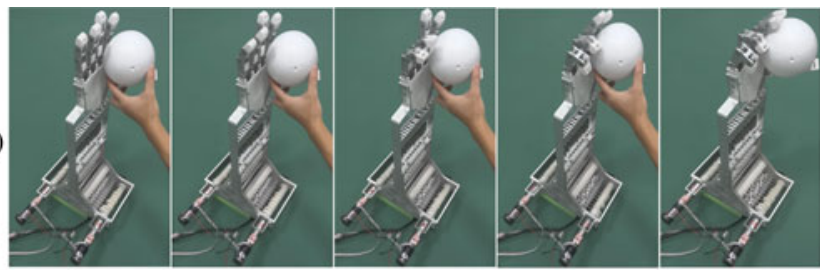

(b)
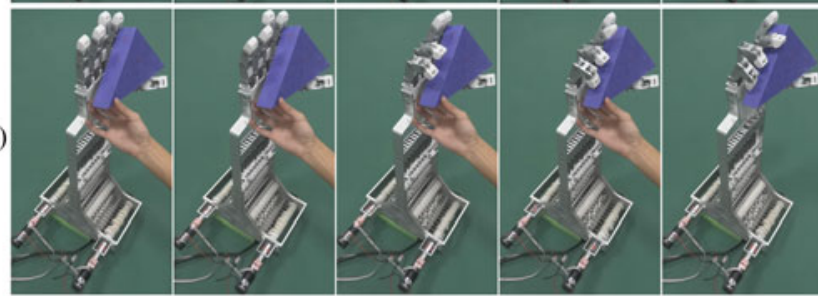

(c)

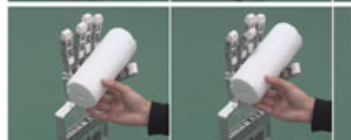

c)

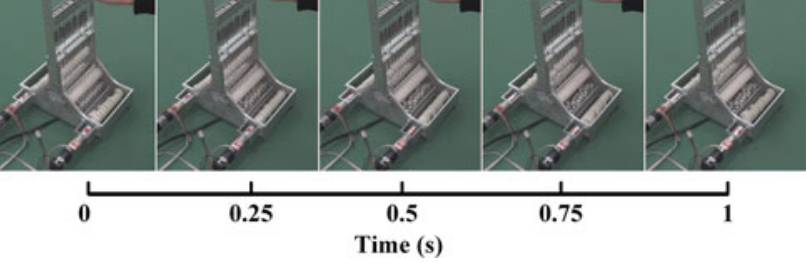

Fig. 19. The temporal posture sequence of the prototype continual grasping three objects in one second: (a) sphere, (b) triangle, and (c) cylinder. The corresponding transmission ratio pairs $\left(K^{1}, K^{2}\right)$ are $(4 / 3,-1.5),(1,1.5)$, and $(5 / 3,1)$, respectively.

simulation, we also give five snapshots averagely sampled along the motion time for each task (see Fig. 19).

When the fingers freely move in space without contacting any objects, the hand posture variation and joints displacement at the end of cam rotation are only dependent on the specified transmission ratio, which is shown in Fig. 18. Thus, the hand posture at the end of the cam rotation is impossible to actively change into other posture in free space for the given transmission ratio. However, if the hand moves in the constrained space, i.e., grasping objects, the final hand posture will not be the posture formed in free space but dependent on the object shapes that are different in scale. This adaptability comes from the used springs implementing soft synergy (see Fig. 13). To illustrate this adaptability, Fig. 20(a) and (b) gives the experiment results in which the hand grasps two different sphere under the same transmission ratio pair $(1 / 3,1)$. Moreover, in order to clearly show the influence of transmission ratio variation on the grasp pattern, we make a minor change of the ratio pair to be $(2 / 3,1)$, the resultant grasp pattern therefore gives some variation on ring and pinky fingers, which is shown in Fig. 20(c).

Additionally, the motion capability of the proposed hand can be generally evaluated by some metrics in the literature [38]. However, for simple operation and quantitative evaluation consideration, the modified relative mean squared error (mRMSE) with dimension free is employed here to evaluate posture difference between robotic hand and human hand at each status along (a)

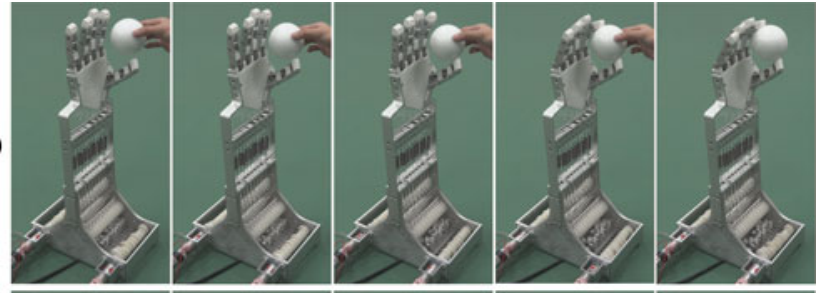

(b)

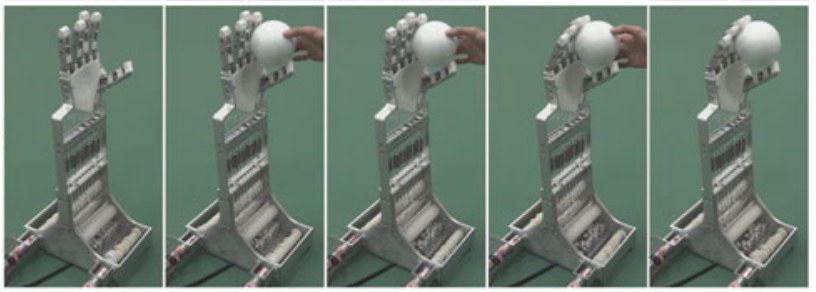

(c)

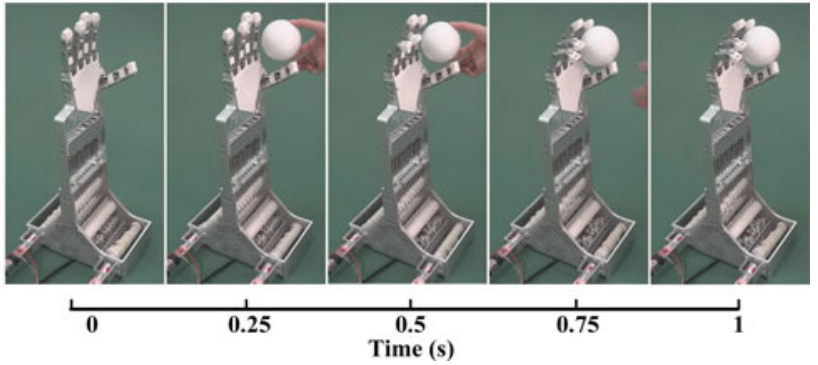

Fig. 20. Temporal sequence of the prototype grasping spheres with different diameter using precision grasp pattern. The transmission ratio pair for grasping sphere in (a) small diameter and (b) big diameter are both $(1 / 3,1)$. After minor changing the ratio pair to $(2 / 3,1)$, the grasp pattern therefore changes to (c).

the motion time

$$
\operatorname{mRMSE}=\sqrt{\frac{1}{n-1} \cdot \frac{\left\|\mathbf{P}_{r d}-\mathbf{P}_{h d}\right\|^{2}}{\left\|\mathbf{P}_{h d}\right\|^{2}}}
$$

where the $\mathbf{P}_{h d}$ and $\mathbf{P}_{r d}$ represent human and robotic hand postures, respectively. The hand posture consisting of the considered joints angle is formulated as vector. The joint angles of robotic hand are measured by the VICON system (Oxford Metrics Ltd, U.K.). To use this evaluation index, the joint data of robotic hand comes from the grasping movements in which no objects are put into the grasp region to ensure the fingers moving freely to replicate the human hand posture. Moreover, in order to avoid the nonzero angle at initial status due to the difference of cable tensioning degree, we use the relative angle variation instead of absolute angle for the evaluation. The four grasping movements shown in Fig. 18 are performed to evaluate the posture difference between the robotic and the human hand along motion time. The corresponding transmission ratio pairs for the robotic hand are specified according to the values shown in Fig. 18. Each grasp trial performed by the robotic hand are evaluated with four grasps performed by four subjects, respectively. Thus, the evaluation result of each grasp corresponds to four error curves. The evaluation results are shown in Fig. 22. As it shown, the posture difference between the robotic hand and human hand among the motion time keep on relatively low level (less than 20\%). The most variation of the error are observed mainly in the first $0.6 \mathrm{~s}$ which covers the mainly flexing stage of the robotic hand fingers (see Fig. 21). Due to the angular 


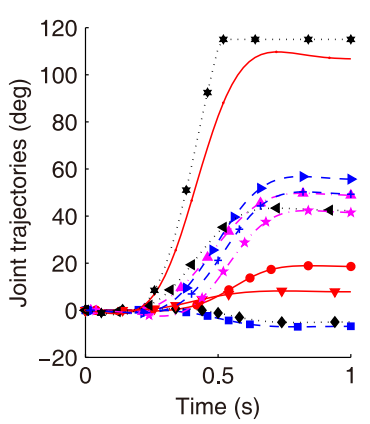

(a)

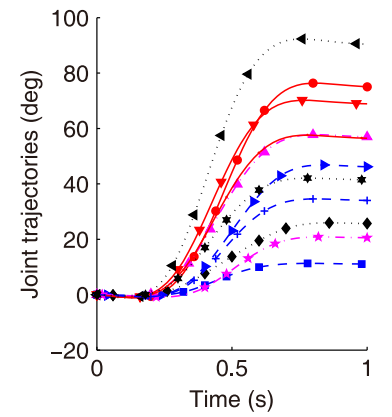

(b)

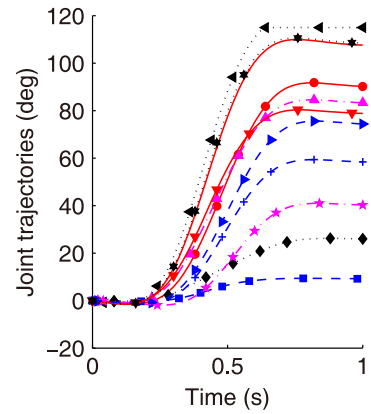

(c)

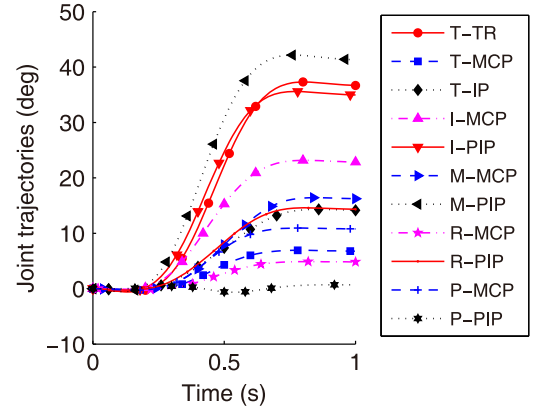

(d)

Fig. 21. The joint trajectories in free space under four transmission ratio pairs: (a) $(4 / 3,-1.5)$, (b) $(1,1.5),($ c) $(5 / 3,1)$, and (d) $(1 / 3,1)$, respectively.

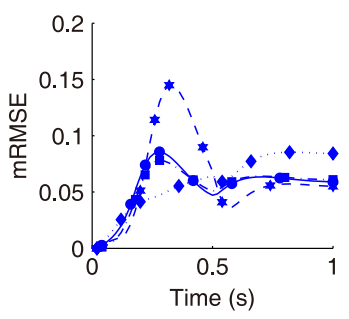

(a)

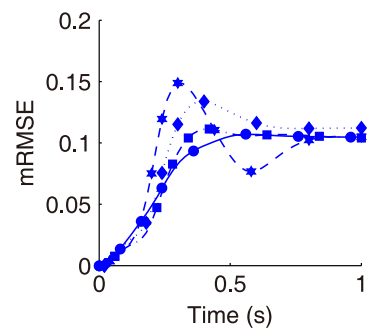

(b)

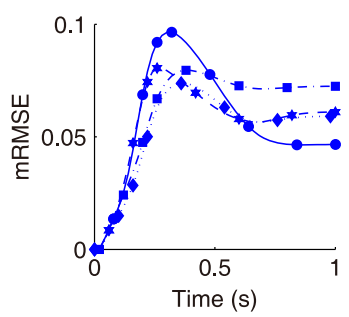

(c)

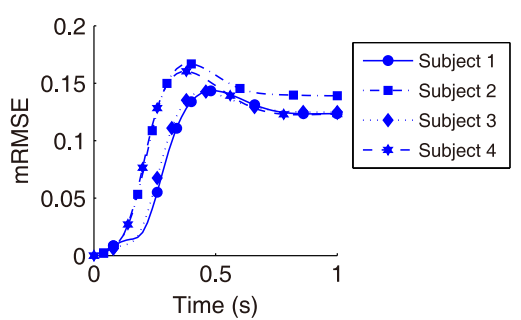

(d)

Fig. 22. Evaluation result of the time-varying posture difference between robotic hand moving in free space and human hand. For each grasp pattern from (a) to (d), the performance of the robotics hand is evaluated by mRMSE with the same grasps performed by four subjects. The correspondence between transmission ratio pair and grasp pattern are: (a) $(4 / 3,-1.5)$ to tripod grasp sphere, (b) $(1,1.5)$ to power grasp triangle, (c) $(5 / 3,1)$ to power grasp cylinder, and $(\mathrm{d})(1 / 3,1)$ to precision grasp bottle with two fingers.

velocity profile discrepancy between the robotic and human hand, the accumulative error increases in the first half of the motion time. This motion reconstruction error mainly originates from the limited number of employed velocity synergies.

However, the simulation and hand grasping experiments validate the proposed mechanical implementation principle of kinematic synergy on generating continual grasping movement. Although the motion period is considered as one second for one cycle in the design framework, the input shafts of the anthropomorphic hand prototype are allowed to rotate fast or slower than $2 \pi \mathrm{rad} / \mathrm{s}$. The motor rotating one cycle in long time gives the slow grasping, whereas the short time brings quick grasping.

\section{DISCUSSION}

\section{A. Cam Design and Grasping Motion Extending}

As shown in Fig. 5, the initial and end velocity of each component in the kinematic synergy is not absolutely equal to zero due to the error in data post procedure. Thus, according to the design formula [see (11)], the corresponded cam radius at $0^{\circ}$ and $360^{\circ}$ may not be the same value. This phenomenon lead to dis-close and nonsmooth contour at the connecting point. However, this problem can be eliminated by locally adjusting the continuity of the contour curve at the connecting point. The geometrical constraints about curve continuity available in the literature of computer graphics, such as equal curvature and equal second-order derivative [39], are applied to the connecting point in parameter space to ensure the continuity and smoothness.

In this paper, the initial posture in fully extending status is specified as the equilibrium state for the kinematic synergy motion synthesis. This design principle is based on mimicking human hand's natural movement. However, the proposed design principle about eigen cam group and ratio regulator can be possibly extended to the object-specific grasping movements in which the grasp postures are considered as equilibrium state. In this case, the underlying human hand motion data comes from the experiment in which the hand moves from one grasping posture instead of the fully extending posture to another grasping posture.

\section{B. Transmission Ratio Distribution and Specification}

According to the distribution of transmission ratio pair shown in Fig. 16, the point near the coordinate axis is generally small and these values are difficult to be implemented using the proposed cable and pulley mechanism because the small ratio requires the small radius of ratio regulator which brings the strength problem. That is why the absolute low limit of the transmission ratio in the previous section is set to 0.3. Actually, 


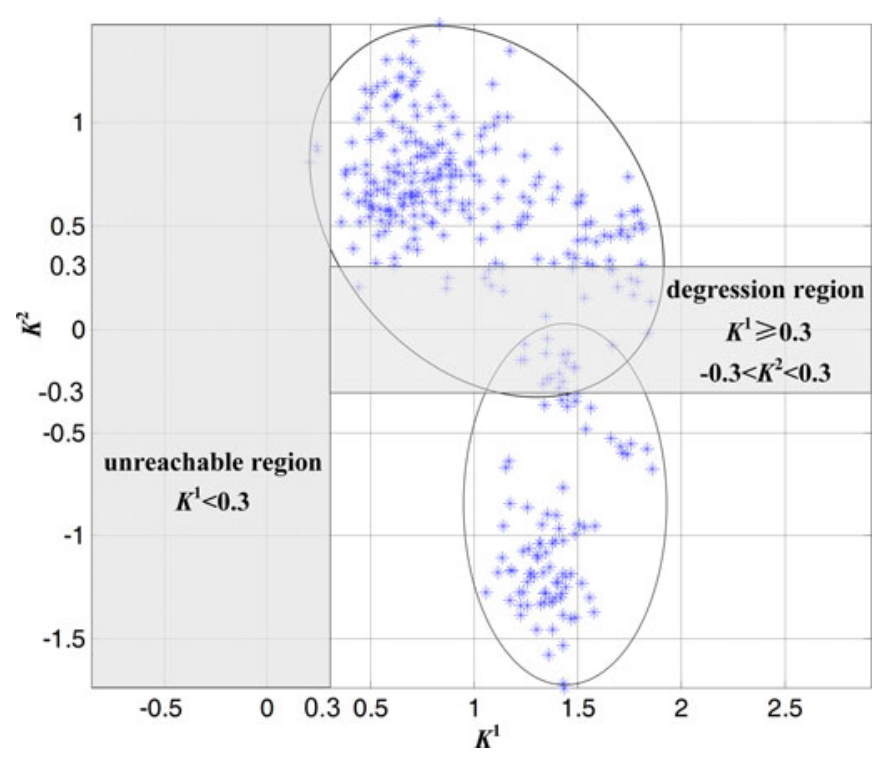

Fig. 23. Unreachable region and degression region on the transmission ratio plane.

the smaller transmission ratio below the absolute low limit has a little effect on the evolvement of hand grasping posture, thus the absolute ratio value below 0.3 can be ignored and set to zero. The zero ratio means the corresponding input shaft of the eigen cam group is motionless during the motion synthesis. Meanwhile, because the second eigen cam group cannot rotate independently and the transmission ratios of the first eigen cam group are always positive for nearly all grasp patterns, it results in the region $\left(K^{1}<0.3\right)$ in the transmission ratio pair plane (Fig. 16) to be unused by the ratio regulator. We call this region the unreachable region (see Fig. 23). Outside the unreachable region, the region in which the transmission ratio for the second eigen cam group degenerates to zero is called the degression region. As shown in Fig. 23, these two regions divide experimental data on the transmission ratio plane into four parts. The ratio pairs, which are outside the unreachable and degression regions, can be freely used to generate proper grasping movement. The ratio pairs in the degression region can only generate the grasping movements that are formed by the first eigen cam group.

In the current version of the anthropomorphic hand prototype, the transmission ratio in each ratio regulator mechanism is manually changed. In fact, two additional motors, such as linear motors, can be employed to automatically adjust the position of the slipping ring in the ratio regulator because all ratio regulators corresponding to one eigen cam group are always set to the same ratio. Thus, what type of command required by these two motors is just the pattern signal corresponding to the transmission ratio pair. The other two motors installed on the input shaft of the eigen cam group can be viewed as an average rotation generator. In such a case, the hand controller first adjusts the transmission ratio to proper value according to the grasp pattern and then drives the two eigen cam groups to averagely rotate one cycle (only the first cam group rotating if the transmission ratio pair falls into the degression region).

\section{Error of Hand Posture Reconstruction}

Only two angular velocity synergies used are the main aspect accounting for the posture discrepancy between the robotic and human hand. More synergies can improve the reconstruction precision of velocity and thus improve the posture precision; however, it will introduce mechanical complexity. Another aspect is the insufficient DoFs of the thumb and the hand cannot make the grasping movement involving thumb rotation and abduction, such as lateral pinch. This limitation can be easily overcome by adding more mechanical DoFs. The long mechanical transmission chain also introduces some error in the posture reconstruction. It can be improved by designing more compact transmission chains. However, there is no doubt about the significance of the proposed principle for designing an anthropomorphic hand and simplifying the control of continual grasping task.

Our work is inspired by the Vinjamuri et al. [18], in which the more general hand movement in a nonfixed onset time is simulated by accumulating the multiweighted time-shifted versions of synergies. In fact, if only considering the movements in normalized motion duration, the simple combination of weighted synergies is acceptable to reconstruct the original velocity.

\section{Compliant Grasp Ability}

The extension springs on each tendon acting as a soft synergy function (see Fig. 13) bring the anthropomorphic hand compliant ability. Before contacting the object, the time-varying posture in free space is determined by the transmission ratio pair. The grasp compliance shows out once any finger contacts the object, especially for the objects whose shape is not consistent with envelope formed by the final grasp posture in free space. For a given transmission ratio pair, the more the inconsistency, the greater the grasp stiffness obtained. Generally, the grasp stiffness is proportional to the stiffness of the employed extension springs. The compliance of our proposed anthropomorphic hand can be considered as one of different features from the MIT hand that is designed based on mechanical implementation of static posture synergy [28].

The additional benefit of the compliant ability is that the appropriate grasp force is introduced after the fingers contact the objects. The evolution of grasp force is dependent on the selected transmission ratio pair, spring stiffness, and object shape. The analysis of static grasp force at each configuration of the underactuated hand with soft synergy is studied in [37]. However, it is a different work to analyze the dynamic grasp force during the continually compliant grasp based on the kinematic synergy. It is viewed as the scope of our future work.

\section{E. Advantage and Potential Applications}

The main contribution of the proposed design principle for the anthropomorphic hand is that the continual grasping movement is only dependent on the selection of transmission ratio pair and no additional procedures, such as computing time-varying weight of each synergy and presetting zero-offset configuration before grasping, are required. The complexity of the control 
strategy is dramatically reduced. The general correspondence between transmission ratio pair and grasp pattern can be constructed by performing quantities of grasp experiment.

The proposed design principle would be particularly useful to design multiDoF myoelectric prosthetic hands in case where the whole drive mechanism would be integrated into the palm. The sEMG signal based pattern recognition and event driven finite-state are prevalently studied to control prosthetic hand multigrasp [35], [40]-[47]. The recognized patterns or events evoked by the input is possibly related to the transmission pair, because the transmission pair in our study and the sEMG pattern both represent some grasp pattern.

The proposed design will also have potential impact on two other application areas. One is designing multifinger endeffector used in an industrial environment or in daily humancentered environments for integrated grasping and manipulation [10]. The second is to construct a humanoid platform with comprehensive grasp ability but easy-to-construct gripper that allows the implementation and tests of various research activities [48], [49].

\section{CONCLUSION}

We have presented a design principle of mechanical implementation of human hand kinematic synergy for continual motion generation of anthropomorphic hand. The principle does not require solving the temporal weight sequence of each synergy and presetting zero-configuration exited in current anthropomorphic hands based on the static posture synergy. Two most significant kinematic synergies observed in angular velocity profile are extracted from quantities of human hand grasping trials and implemented by the two eigen cam groups, respectively. The continual grasping of the anthropomorphic hand is only dependent on prespecifying the transmission ratio pair and the input motors just need to rotate averagely one cycle during the motion time.

The grasping simulation and experiment of the developed prototype demonstrate the continual grasping ability under different transmission ratio pairs. We also give the time-varying posture evolvement law of each single kinematic synergy. The distribution of transmission ratio pair corresponding to the feasible grasp patterns of the prototype hand is also elaborated.

The potential application of the design principle is the prosthetic hand whose control source is pattern signal from sEMG. We are currently developing a comprehensive anthropomorphic hand toward improving the grasping quality and implementing sense feedback by adding more mechanical DoFs and tactile sensors. Additionally, designing a more compact drive mechanism for integration into the hand palm will give the hand more particular utility. It is also viewed as the scope of our future work.

\section{REFERENCES}

[1] Z. Ju and H. Liu, "Human hand motion analysis with multisensory information," IEEE/ASME Trans. Mechatronics, vol. 19, no. 2, pp. 456-466, Apr. 2014

[2] E. Rombokas, M. Malhotra, E. A. Theodorou, E. Todorov, and Y. Matsuoka, "Reinforcement learning and synergistic control of the ACT hand," IEEE/ASME Trans. Mechatronics, vol. 18, no. 2, pp. 569-577, Apr. 2013.

[3] W. Townsend, "The barretthand grasper-programmably flexible part handling and assembly," Ind. Robot, vol. 27, no. 3, pp. 181-188, Apr. 2000.

[4] N. Fukaya, T. Asfour, R. Dillmann, and S. Toyama, "Development of a five-finger dexterous hand without feedback control: The TUAT/Karlsruhe humanoid hand," in Proc. IEEE/RSJ Int. Conf. Intell. Robot. Syst., Tokyo, Japan, 2013, pp. 4533-4540.

[5] N. Dechev, W. L. Cleghorn, and S. Naumann, "Multiple finger, passive adaptive grasp prosthetic hand," Mech. Mach. Theory, vol. 36, no. 10, pp. 1157-1173, Oct. 2001.

[6] D. Aukes, S. Kim, P. Garcia, A. Edsinger, and M. R. Cutkosky, "Selectively compliant underactuated hand for mobile manipulation," in Proc. IEEE Int. Conf. Robot. Autom., 2012, pp. 2824-2829.

[7] A. M. Dollar and R. D. Howe, "A robust compliant grasper via shape deposition manufacturing," IEEE/ASME Trans. Mechatronics, vol. 11, no. 2, pp. 154-161, Apr. 2006.

[8] C. Gosselin, F. Pelletier, and T. Laliberte, "An anthropomorphic underactuated robotic hand with 15 dofs and a single actuator," in Proc. IEEE Int. Conf. Robot. Autom., 2008, pp. 749-754.

[9] A. Bierbaum, J. Schill, T. Asfour, and R. Dillmann, "Force position control for a pneumatic anthropomorphic hand," in Proc. IEEE/RAS Int. Conf. Humanoid Robot., Paris, France, 2009, pp. 21-27.

[10] I. Gaiser, S. Schulz, A. Kargov, H. Klosek, A. Bierbaum, C. Pylatiuk, R. Oberle, T. Werner, T. Asfour, G. Bretthauer, and R. Dillmann, "A new anthropomorphic robotic hand," in Proc. IEEE/RAS Int. Conf. Humanoid Robot., Daejeon, Korea, 2008, pp. 418-422.

[11] S. Schulz, C. Pylatiuk, and G. Bretthauer, "A new ultralight anthropomorphic hand," in Proc. IEEE Int. Conf. Robot. Autom., Seoul, Korea, 2001, pp. 2437-2441

[12] L. Birglen, T. Laliberté, and C. Gosselin, "Grasping vs. manipulating," in Underactuated Robotic Hands, Springer Tracts in Advanced Robotics, B. Siciliano, O. Khatib, and F. Groen, eds., Berlin, Germany: Springer, 2008, vol. 40, pp. 7-31.

[13] J. Romero, T. Feix, C. H. Ek, H. Kjellstrom, and D. Kragic, "Extracting postural synergies for robotic grasping," IEEE Trans. Robot., vol. 29, no. 6, pp. 1342-1352, Dec. 2013.

[14] J. Steffen, R. Haschke, and H. Ritter, "Towards dextrous manipulation using manipulation manifolds," in Proc. IEEE/RSJ Int. Conf. Robot. Intell. Syst., Nice, France, 2008, pp. 2738-2743.

[15] M. Santello, M. Flanders, and J. F. Soechting, "Postural hand synergies for tool use," J. Neurosci., vol. 18, no. 23, pp. 10105-10115, Dec. 1998.

[16] C. Mason, J. Gomez, and T. Ebner, "Hand synergies during reach-tograsp," J. Neurophysiol., vol. 86, no. 6, pp. 2896-2910, Dec. 2001.

[17] I. V. Grinyagin, E. V. Biryukova, and M. A. Maier, "Kinematic and dynamic synergies of human precision-grip movements," J. Neurophysiol., vol. 94, no. 4, pp. 2284-2294, Oct. 2005.

[18] R. Vinjamuri, S. Mingui, C. Cheng-Chun, L. Heung-No, R. J. Sclabassi, and M. Zhi-Hong, "Dimensionality reduction in control and coordination of the human hand," IEEE Trans. Biomed. Eng., vol. 57, no. 2, pp. 284-295, Feb. 2010.

[19] M. Santello, M. Flanders, and J. F. Soechting, "Patterns of hand motion during grasping and the influence of sensory guidance," J. Neurosci. vol. 22, no. 4, pp. 1426-1435, Feb. 2002.

[20] R. Vinjamuri, M. Sun, C.-C. Chang, H.-N. Lee, R. J. Sclabassi, and Z.-H. Mao, "Temporal postural synergies of the hand in rapid grasping tasks," IEEE T. Inf. Technol. Biomed., vol. 14, no. 4, pp. 986-994, Jul. 2010

[21] C. Castellini and P. van der Smagt, "Evidence of muscle synergies during human grasping," Biol. Cybern., vol. 107, no. 2, pp. 233-245, Apr. 2013.

[22] N. Karnati, B. A. Kent, and E. D. Engeberg, "Bioinspired sinusoidal finger joint synergies for a dexterous robotic hand to screw and unscrew objects with different diameters," IEEE/ASME Trans. Mechatronics, vol. 18, no. 2, pp. 612-623, Apr. 2013.

[23] E. Rombokas, M. Malhotra, and Y. Matsuoka, "Task-specific demonstration and practiced synergies for writing with the ACT hand," in Proc. IEEE Int. Conf. Robot. Autom., Shanghai, China, 2011, pp. 5363-5368.

[24] A. Zhang, M. Malhotra, and Y. Matsuoka, "Musical piano performance by the ACT Hand," in Proc. IEEE Int. Conf. Robot. Autom., Shanghai, China, 2011, pp. 3536-3541

[25] T. Wimbock, B. Jahn, and G. Hirzinger, "Synergy level impedance control for multifingered hands," in Proc. IEEE/RSJ Int. Conf. Intell. Robot. Syst., 2011, pp. 973-979.

[26] J. Rosell, R. Suárez, C. Rosales, and A. Pérez, "Autonomous motion planning of a hand-arm robotic system based on captured human-like 
hand postures," Auton. Robot., vol. 31, no. 1, pp. 87-102, Jul. 2011.

[27] F. Ficuciello, G. Palli, C. Melchiorri, and B. Siciliano, "Experimental evaluation of postural synergies during reach to grasp with the UB hand IV," in Proc. IEEE/RSJ Int. Conf. Intell. Robot. Syst., 2011, pp. 17751780.

[28] C. Y. Brown and H. H. Asada, "Inter-finger coordination and postural synergies in robot hands via mechanical implementation of principal components analysis," in Proc. IEEE/RSJ Int. Conf. Intell. Robot. Syst., San Diego, CA, USA, 2007, pp. 2877-2882.

[29] X. Kai, D. Yuheng, L. Huan, S. Xinjun, and Z. Xiangyang, "Mechanical implementation of postural synergies of an underactuated prosthetic hand," in Proc. 6th Int. Conf. Intell. Robot. Appl., Busan, South Korea, 2013, pp. $463-474$.

[30] X. Kai, Z. Jiangran, D. Yuheng, S. Xinjun, and Z. Xiangyang, "Design and postural synergy synthesis of a prosthetic hand for a manipulation task," in Proc. IEEE/ASME Int. Conf. Adv. Intell. Mechatronics, Novotel Wollongong, Australia, 2013, pp. 56-62.

[31] G. Matrone, C. Cipriani, E. Secco, G. Magenes, and M. Carrozza, "Principal components analysis based control of a multi-dof underactuated prosthetic hand," J. Neuroeng. Rehabil., vol. 7, no. 16, pp. 1-13, Apr. 2010.

[32] G. Matrone, C. Cipriani, M. Carrozza, and G. Magenes, "Real-time myoelectric control of a multi-fingered hand prosthesis using principal components analysis," J. Neuroeng. Rehabil., vol. 9, no. 40, pp. 1-13, Jun. 2012.

[33] M. R. Cutkosky, "On grasp choice, grasp models, and the design of hands for manufacturing tasks," IEEE Trans. Robot. Autom., vol. 5, no. 3, pp. 269-279, Jun. 1989.

[34] B. Yi, F. C. Harris Jr., L. Wang, and Y. Yan, "Real-time natural hand gestures," Comput. Sci. Eng., vol. 7, no. 3, pp. 92-96, May 2005.

[35] C. Cipriani, C. Antfolk, M. Controzzi, G. Lundborg, B. Rosen, M. C. Carrozza, and F. Sebelius, "Online myoelectric control of a dexterous hand prosthesis by transradial amputees," IEEE Trans. Neural Syst. Rehabil. Eng., vol. 19, no. 3, pp. 260-270, Jun. 2011.

[36] A. Bicchi, M. Gabiccini, and M. Santello, "Modelling natural and artificial hands with synergies," Philos. Trans. R. Soc. B, Biol. Sci., vol. 366, no. 1581, pp. 3153-3161, Nov. 2011.

[37] G. Grioli, M. Catalano, E. Silvestro, S. Tono, and A. Bicchi, "Adaptive synergies: an approach to the design of under-actuated robotic hands," in Proc. IEEE/RSJ Int. Conf. Intell. Robot. Syst., Vilamoura, Portugal, 2012, pp. 1251-1256.

[38] T. Feix, J. Romero, C. H. Ek, H. B. Schmiedmayer, and D. Kragic, "A metric for comparing the anthropomorphic motion capability of artificial hands," IEEE Trans. Robot., vol. 29, no. 1, pp. 82-93, Feb. 2013.

[39] L. A. Piegl and W. Tiller, The NURBS Book, 2nd ed., Berlin, Germany: Springer, 1997, pp. 80-120.

[40] G. Li, A. E. Schultz, and T. A. Kuiken, "Quantifying pattern recognitionbased myoelectric control of multifunctional transradial prostheses," IEEE Trans. Neural Syst. Rehabil. Eng., vol. 18, no. 2, pp. 185-192, Apr. 2010.

[41] C. Jun-Uk, M. Inhyuk, and M. Mu-seong, "A real-time EMG pattern recognition system based on linear-nonlinear feature projection for a multifunction myoelectric hand," IEEE Trans. Biomed. Eng., vol. 53, no. 11, pp. 2232-2239, Nov. 2006.

[42] F. C. Sebelius, B. N. Rosen, and G. N. Lundborg, "Refined myoelectric control in below-elbow amputees using artificial neural networks and a data glove," J. Hand Surg., vol. 30, no. 4, pp. 780-789, Jul. 2005.

[43] L. H. Smith, L. J. Hargrove, B. A. Lock, and T. A. Kuiken, "Determining the optimal window length for pattern recognition-based myoelectric control: Balancing the competing effects of classification error and controller delay," IEEE Trans. Neural Syst. Rehabil. Eng., vol. 19, no. 2, pp. 186-192, Apr. 2011.

[44] L. Hargrove, Y. Losier, B. Lock, K. Englehart, and B. Hudgins, "A real-time pattern recognition based myoelectric control usability study implemented in a virtual environment," in Proc. IEEE Int. Conf. Eng. Med. Biol. Soc., Lyon, France, 2007, pp. 4842-4845.

[45] S. A. Dalley, H. A. Varol, and M. Goldfarb, "A method for the control of multigrasp myoelectric prosthetic hands," IEEE Trans. Neural Syst. Rehabil. Eng., vol. 20, no. 1, pp. 58-67, Jan. 2012.

[46] S. Micera, J. Carpaneto, and S. Raspopovic, "Control of hand prostheses using peripheral information," IEEE Rev. Biomed. Eng., vol. 3, pp. 48-68, Dec. 2010.
[47] R. G. Clement, K. E. Bugler, and C. W. Oliver, "Bionic prosthetic hands: A review of present technology and future aspirations," Surg. J. R. Coll. Surg. Edinb. Irel., vol. 9, no. 6, pp. 336-340, Dec. 2011.

[48] W. Chen and C. Xiong, "A principle of mechanical implementing the kinematic synergy for designing anthropomorphic hand," in Proc. 6th Int Conf. Intell. Robot. Appl., Busan, South Korea, 2013, pp. 339-350.

[49] F. Rothling, R. Haschke, J. J. Steil, and H. Ritter, "Platform portable anthropomorphic grasping with the bielefeld 20-DOF shadow and 9-DOF TUM hand," in Proc. IEEE/RSJ Int. Conf. Intell. Robot. Syst., San Diego, CA, USA, 2007, pp. 2951-2956.

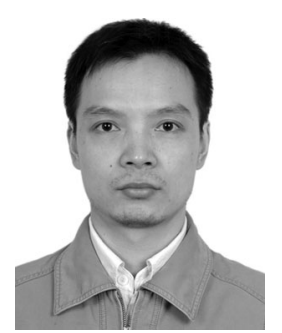

Wenbin Chen (M'12) received the B.S. degree in mechanical engineering from Wuhan University of Science and Technology, Wuhan, China, in 2004, and the Ph.D. degree in mechatronic engineering from Huazhong University of Science and Technology (HUST), Wuhan, China, in 2012.

He is currently a Postdoctoral Fellow in the State Key Laboratory of Digital Manufacturing and Equipment Technology, HUST. He is also a Research Fellow of Computer Science at the Lincoln School of Computer Science, University of Lincoln, Lincoln, U.K. His research interests include anthropomorphic prosthetic robotics, rehabilitation robotics, and robot motion planning and control.

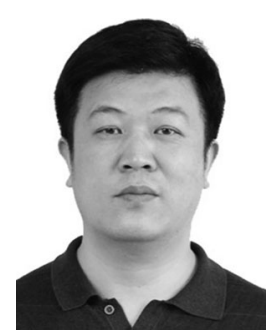

Caihua Xiong (M'12) received the Ph.D. degree in mechanical engineering from Huazhong University of Science and Technology (HUST), Wuhan, China, in 1998.

From 1999 to 2003, he was with the City University of Hong Kong and the Chinese University of Hong Kong, Hong Kong, as a Postdoctoral Fellow, and Worcester Polytechnic Institute, Worcester, MA, USA, as a Research Scientist. He has been a Chang Jiang Professor at HUST since 2008. His current research interests include biomechatronic prostheses, rehabilitation robotics, and robot motion planning and control. He is also the Director of the Institute of Rehabilitation and Medical Robotics at HUST.

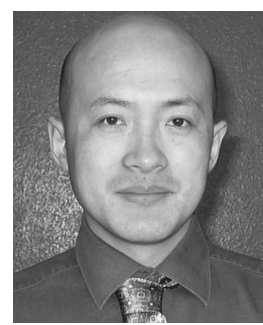

Shigang Yue (M'05) received the Ph.D. degree from Beijing University of Technology, Beijing, China, in 1996.

He is currently a Professor of Computer Science at the Lincoln School of Computer Science, University of Lincoln, Lincoln, U.K. He was an Alexander von Humboldt Research Fellow from 2000 to 2001 at the University of Kaiserslautern, Kaiserslautern, Germany. Before joining the University of Lincoln in 2012, he held research positions at the University of Cambridge, Newcastle University, and University College London. He is the founding Director of the Computational Intelligence Laboratory at the University of Lincoln and the Coordinator for several EU FP7 projects. His research interests include artificial intelligence, computer vision, robotics, brains, and neuroscience.

Dr. Yue is a member of the International Neural Network Society, the International Society for Artificial Life, and the International Society for Behavioral Ecology. 